\title{
La vie politique djiboutienne de 1992 à 2021 : quelques réflexions sur le changement et les réformes politiques.
}

Résumé: Depuis 1992 le modèle de changement politique retenu par les autorités politiques djiboutiennes n'a pas aboutit à une alternance au pouvoir. L'introduction de la compétition électorale ni l'apparition d'une Commission Electorale Nationale Indépendante en 2002 n'ont aboutit à l'apparition d'un régime politique où l'opposition dispose d'un statut.

Les réformes constitutionnelles ultérieures et surtout la suppression de la norme limitant les mandats présidentiels en 2010 ont renforcé plus le pouvoir du président qu'une réelle évolution du système vers un régime où il y a la libre expression et le respect de l'opposition politique, qui a eu ses premiers députés lors des dernières élections législatives en février dernier. Cependant la situation socioéconomique a beaucoup changé avec l'accroissement des investissements étrangers, provenant des pays d'Asie et du monde arabe par la transformation du Port de Djibouti en hub régional.

Mots clés : Changement politique, processus, réforme, opposition,

Resumo: Desde 1992, o modelo de políticas de mudanças tomadas pelas autoridades de Djibouti não levaram a uma mudança no poder. A introdução da competição eleitoral ou o desenvolvimento de uma Comissão Eleitoral Nacional Independente em 2002, elevou ao surgimento de um sistema politico onde a oposição não tem um status.

As reformas constitucionais posteriores, especialmente, a remoção da norma limitando o mandato presidencial em 2010 têm fortalecido o poder do presidente mais do que uma evolução política, teve seus primeiros membros nas últimas eleições parlamentares, em fevereiro de 2013. No entanto, a situação 
La vie politique djiboutienne de 1992 a 2021 : quelques reflexions sur le changement et les reformes politiques

socio-econômica mudou drasticamente como aumento do investimento estrangeiro de países asiáticos e do mundo árabe, tranformando o Porto de Djibouti um pólo regional.

Palavras chaves: cambio político, proceso, reforma, oposição

\section{INTRODUCTION}

En octobre 2020, la question du troisième mandat présidentiel a surgit sur la scène politique de beaucoup de pays et en particulier les pays francophones d'Afrique de l'Ouest'. Or la limitation à deux mandats présidentiels a été novateur dans le paysage Plus de trois décennies de la vague de changement constitutionnel ou de transition démocratique des pays africains subsahariens, les révisions frénétiques des constitutions libérales des années 1990 et les coups d'Etat en Centrafrique en mars 2013 et au Mali en 2020 soulèvent la question de la consolidation du changement politique. C'est un désaveu des analyses des africanistes africains.es et occidentaux.es, qui avaient prématurément misé sur une transition vers des régimes politiques pluralistes et libéraux, donc démocratiques. Ils/elles avaient pris en considération deux éléments, importants certes, mais qu'on ne pourrait réduire à des cultures et des réalités complexes. La fin des régimes autoritaires au début de l'année 2000 fut-elle une simple parenthèse ? Ou une modernisation de l'autoritarisme des régimes postcoloniaux d'Afrique?

L'objet de cette réflexion est de faire un bilan critique du processus de changement politique et socioéconomique de la République de Djibouti, qui a débuté en septembre 1992, après une brève période de «libéralisation politique ${ }^{2}$.» L'expression «changement politique» est prise ici au sens de

\footnotetext{
Bahdon Mohamed Abdillahi, Opposition, société civile et troisième mandat présidentiel en Afrique de l'Ouest, Afriqueducation, ${ }^{\circ} 490$, octobre 2020, pp. 16-17. Loada Augustin, La limitation du nombre des mandats présidentiels en Afrique francophones, Afrilex, $\mathrm{n}^{\circ}$ 03, 2003, in http://afrilex.u-bordeaux.fr/wp-content/uploads/2021/03/3doc8loada.pdf

2 Nous reprenons ici le sens donné par le politologue argentin Guillermo O’Donnell, la libéralisation politique est une période charnière entre le début de la fin du régime autoritaire et la préparation du nouveau régime. Un des éléments visibles de cette période est la baisse de la violence politique et la reconnaissance l'opposition. Guillermo O'Donnell, Philippe C. Schmitter, and Laurence Whitehead, Transitions from Authoritarian Rule. Southern Europe, Johns Hopkins University Press, Baltimore, 1986.
} 
changement de régime suivant l'analyse de Morlino (1985 : 30) pour qui le «changement politique est un changement de ou du système politique.» Pour lui, le changement politique n'est autre qu'une transformation que subit un système ou ses composants par l'introduction de nouvelles institutions politiques. D'autres auteurs comme Quantin, Patrick et al (1996) ou Comi Toulabor (1991) utilisent le terme «transition» au lieu de changement. Le recours aux termes peut prêter parfois à confusion. Dans «transition» les analystes politiques prédéterminent la phase finale de la période dite de transition vers le régime démocratique alors que le changement est un fait qui intervient dans tout régime politique et suivant Morlino il est peut être continu ou discontinu, pacifique ou violent, fondamental ou marginal, accéléré ou lent, innovateur ou non innovateur... Dans le cas de certains régimes politiques africains, ce qui s'est produit, c'est plutôt un changement des formes comme la relative importance donnée à des élections compétitives et des passations pacifiques du pouvoir présidentiel dans quelques rares exemples comme le Bénin, le Bostwana, le Ghana, le Mali (avant le Coup d'Etat de mars 2012) et les Iles Capes Verts.

Cependant que ça soit la transition ou le changement, les valeurs et les règles du jeu changent; il est évident que des régimes différents sur le plan philosophique ne sont pas régis par les mêmes règles. Une analyse essentiellement juridique fait ressortir l'importance de ces règles dans le système politique alors qu'une analyse politique met l'accent sur des facteurs sociologiques et donc de l'opposition de groupes organisés pour conquérir le pouvoir, lesquels recourent non seulement aux règles, mais aussi l'utilisation d'autres ressources sociales ou la violence comme on a pu observer au Kenya en 2007.

Cette analyse s'articule autour de deux principaux points d'une part il s'agit d'analyser le contexte, qui est à la base du processus, c'est-à-dire le contexte sociopolitique de l'année 1991 et d'autre part l'introduction de la compétition électorale et ses conséquences sur tant sur la distribution des pouvoirs politiques que sur l'alternance au sommet de l'Etat. 
La vie politique djiboutienne de 1992 a 2021 : quelques reflexions sur le changement et les reformes politiques

\section{LE CHANGEMENT POLITIQUE DANS UN CONTEXTE D'INSTABILITÉ RÉGIONALE ET DES} CONTESTATIONS POLITIQUES NATIONALES

Pour analyser et comprendre le changement politique de la République de Djibouti, l'analyse du contexte politique national et régional s'impose. En d'autres termes et en reprenant l'analyse de Morlino (1985: 82), il s'agit ici d'analyser le "pourquoi" du changement. Le pourquoi est aussi important que «le comment» comme nous allons voir postérieurement.

A la base de tout changement social et/ou politique, on note l'impact de facteurs internes et externes (Fukuyama, 1992, Pridham, 1994, Zolberg, 1985, ) sur le système sociopolitique en question ; les uns sont un produit qui résulte de la dynamique des forces sociales internes et les autres des relations internationales ou par l'imposition de ceux qui dominent le système international. Au début de la décennie 1990 ces facteurs sont tellement importants ; ils ont un impact décisif sur les systèmes politiques de beaucoup de pays ayant des régimes autoritaires ou communistes, sauf Cuba et la Corée du Nord. En République de Djibouti, on trouve les mêmes facteurs internes que les autres pays africains : une croissance économique en net recul, augmentation de l'endettement et la raréfaction des ressources du régime, donc diminution de la capacité distributive des ressources pour son maintien ou sa survie. Mais ils ne sont pas appropriés par la population et une opposition locale, il n'y a pas de grands mouvements populaires dans les grandes villes et dans la capitale comme dans d'autres pays africains. Ce qui conduit au président de la République Hassan Gouled Aptidon à minimiser l'intérêt du changement politique que certains pays du continent se sont engagés, changement qu'il

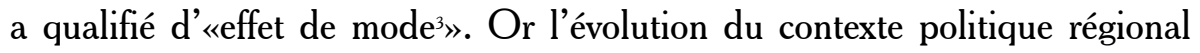
va être décisive sur la situation politique nationale. Ce contexte est marqué depuis trois décennies par les guerres civiles et des guerres étatiques. Mais deux événements politiques et sociaux vont avoir un impact important sur la situation djiboutienne : la fin des deux régimes militaires de la Somalie et de l'Ethiopie l'année 1991 et le nouveau discours de la France - qui lie sa coopération aux réformes démocratiques qu'entreprennent les pays africains.

Comme affirme Roland $\mathrm{Marshal}^{4}$, la fin de ces deux régimes est une au-

$4 \quad$ R. Marshall écrit «qu' en l'espace de quelques mois, plusieurs difficultés qui avaient miné les tentatives de luttes armées vers la fin des années 1970 (lors de la constitution du Front démocratique de libération de 
África (São Paulo, 1978, Online), São Paulo, n. 42, p. 177-219, 2021

baine pour le mouvement rebelle afar djiboutien quant à la facilité qui s'offrait à lui pour mener des attaques contre le pouvoir central ${ }^{5}$. Le contexte politique de la Corne de l'Afrique contraste avec le reste du continent africain et surtout l'Afrique de l'Ouest et du Centre où les changements politiques s'engagent pacifiquement, même si par la suite des guerres civiles éclatent comme au Congo. En République de Djibouti la conjugaison d'une situation régionale violente de fin des régimes en Ethiopie et en Somalie et l'éclatement d'une guerre civile djiboutienne constituent paradoxalement le déclenchement d'un processus de changement politique.

\subsection{LA GUERRE CIVILE DE 1991 COMME FACTEUR DÉCLENCHEUR DU CHANGEMENT POLITIQUE}

Dans ce paragraphe il s'agit d'analyser «le comment» et "le quand", c'est-à-dire la date à laquelle commence ledit changement. Comment changer est aussi important, parce que sans la pression, l'adhésion et la participation de tous les acteurs adhèrent au changement, on n'arrive pas à une amorce d'un changement du jeu politique au moins sur le plan constitutionnel et juridique.

L'année 1991 est une date importante dans l'histoire politique récente de la République de Djibouti. En effet, c'est l'année où éclate une guerre civile, la première du genre dans ce petit pays de la Corne de l'Afrique, connu comme un Oasis de Paix dans une région instable depuis les années 1960. Des affrontements armés opposent entre les forces gouvernementales et un front armé, le Front pour la Restauration de l'Unité et de la Démocratie (FRUD) ${ }^{6}$ dans le pays afar à partir d'octobre 1991. Ces affrontements

Djibouti) étaient levées : l'armement était disponible en quantité et en qualité; l'effondrement de l'appareil d'Etat éthiopien permettait la constitution d'un sanctuaire accessible sans condition politique forte, la dissolution de la milice afar de la région autonome d'Assab offrait de réelles possibilités de recrutement de cadres expérimentés» in «La fin du régime de Mengistu et la situation de la Corne de l'Afrique, L'après Mengistu dans la Corne de l'Afrique», Relations Internationales, 1993.

5 Elle est mise en pratique dès le début de l'année 1990 par des petits fronts rebelles qui attaquent des postes militaires frontaliers au Nord en pays afar, sans que cela préoccupe pour autant le pouvoir djiboutien. Ces attaques continuent et aboutissent finalement à des affrontements plus intenses entre un groupe de rebelles et les forces gouvernementales dans cette partie du pays.

6 C'est un groupe d'agrégation de fronts animés par des Afars. Certains existent depuis plus de 20 ans. Voir A. Coubba, Djibouti : une nation en otage, Paris, L'Harmattan, 1993. Mohamed Kadamy, ancien 
La vie politique djiboutienne de 1992 a 2021 : quelques reflexions sur le changement et les reformes politiques

mettent à jour une opposition au pouvoir central, qui n’a jamais disparu, malgré l'affirmation du président de la République en avril 1987 de l'inexistence d'une opposition à son gouvernement. Le Mouvement Populaire de Libération (MPL) et le Front Démocratique de Libération de Djibouti (MDLD), présents sur la scène politique nationale dans les années 1960 et 1970, reconstituent leurs appareils militaires et politiques dans l'ombre. Analysant la situation à laquelle la République de Djibouti est confrontée, Gabriel Lamarche (1993) affirme que le «pouvoir djiboutien, qui avait toujours refusé de prendre en compte ces facteurs de crise, se trouva confronté, dès les premiers mois de 1991, à un premier mouvement armé, l'Action pour la Révision de l'Ordre à Djibouti (AROD)». Les mouvements afar se regroupent autour d'un front puissant dénommé Front pour la Restauration de l'Unité et de la Démocratie (FRUD)7 ${ }^{7}$, dirigé par le premier Premier ministre du pays, Ahmed Dini Ahmed. L'une des demandes de ce front rebelle est la restauration de la démocratie. En fait il s'agit d'instaurer la démocratie dans ce pays. Il faut rappeler que le pays a connu à partir de 1981 l'institutionnalisation du régime de parti unique et donc le refus de toute opposition politique.

Ces affrontements armés occultent une prise de position politique d'un membre de l'ex parti unique, le Rassemblement Populaire pour le Progrès (RPP) le député, feu Mohamed Ahmed Issa. En effet en mars 1990, celui-ci propose au comité central du parti gouvernemental le RPP “l'instauration de la démocratie à l'instar de ce qui se passe dans les autres pays du continent noir $»^{8}$. Cette proposition est rejetée par les membres de cet organe dirigé par le Président de la République en sa qualité de Président du parti. L'intérêt de la proposition montre au moins la fin d'un consensus de façade autour de la politique du Chef de l'Etat dont sa position faisait autorité pour toute la classe

porte-parole du Frud en Europe, arrêté en Ethiopie et emprisonné à Djibouti expliquait en ces termes le Frud : «c'est une organisation politico-militaire qui est issue d'un long processus. Il est le fruit de toutes les luttes multiformes qui se sont déroulées depuis l'indépendance. On peut même considérer que c'est un continuum historique des mouvements qui ont existé comme le Mouvement populaire pour la libération (MPL), le Front démocratique pour la libération de Djibouti (FDLD), le Parti populaire djiboutien (PPD), l'Union des mouvements démocratiques (UMD) et qui, à partir du 12 août 1991, ont pris la forme du Front pour la restauration de l'unité et de la démocratie". In Interview à Nouvelles d'Addis du 27 octobre 2000, www. lesnouvelleaddis.org, consulté le 15 décembre 2004.

Ali Coubba, Op. cit.

$8 \quad$ Lettre de l'Océan Indien du 27 mars 1990. Il signe le 17 février avec d'autres personnalités un Manifeste national favorable au multipartisme. 
politique. Mais au-delà de l'objet de la proposition, c'est une autre manière de faire la politique en reconnaissant l'existence d'autres forces politiques et donc le dialogue avec celles-là qui est présenté. Or son rejet conduit à une certaine radicalisation de l'aile dure du régime, qui a privilégiée la voie militaire contre les groupes armés ${ }^{9}$. Pour lui, le FRUD représente des intérêts étrangers, autrement dit c'est une organisation étrangère. Dans ses discours, il ne cesse de parler de «bandits» à propos des rebelles afars pour convaincre la France d'intervenir militairement, mais celle-ci refuse l'application de ces accords en arguant, d'après Marshall (1995), que «la rébellion afar était une affaire interne». Cette attitude du gouvernement français est perçue comme un signe de changement symbolique dans les relations avec certains régimes politiques africains. Mais suite à ce refus, une crise naît entre les deux capitales. Le président djiboutien durcit le ton surtout pour la suspension de l'aide budgétaire française et accuse un soutien indirect des militaires français, déployés sur le terrain, aux rebelles du FRUD. Selon Lemarche la France suspend l'activité de ses coopérants. Selon ce dernier c'est «un signal politique aux autorités djiboutiennes». Ces dernières ignorèrent cette position.

Les affrontements armés, qui s’intensifient, ont des conséquences politiques importantes. Ils provoquent une crise gouvernementale; le 14 janvier 1992 le ministre de la Santé et des Affaires Sociales, Mohamed Djama Elabé, démissionne, suivi par un autre ministre, celui de la Fonction Publique et des Réformes Administratives et 14 députés, donc une vingtaine des parlementaires (certains ministres démissionnaires sont aussi des parlementaires) sur 65 au total constituent une «opposition parlementaire», c'est l'expression donnée à ce groupe à l'Assemblée Nationale. Mais le pouvoir djiboutien durcit le ton. Cependant ce contexte sociopolitique renforce non seulement la position des contestataires de l'intérieur, ex membres du gouvernement et députés à l'Assemblée Nationale comme l'initiateur de ladite proposition d'ouverture politique, Mohamed Ahmed Issa, mais aussi ceux de l'extérieur notamment les exilés qui vivent en Europe. L'émergence ${ }^{10}$ de ce front sur la

\footnotetext{
Aucune négociation ne s'engage entre les deux parties. Le Président de la République se tourne quelques mois avant l'indépendance en 1977.

10 Ebo Houmed Alwane, «Djibouti, Tensions sociopolitiques sur fond de succession», L'Afrique Politique Revendications populaires et recompositions politiques», Paris, Karthala, 1997, pp.85-110.
} 
La vie politique djiboutienne de 1992 a 2021 : quelques reflexions sur le changement et les reformes politiques

scène politique nationale ouvre une brèche dans le monolithisme politique instauré par un homme et sa formation politique, seule formation politique reconnue dans le pays depuis 1981, date à laquelle elle est institutionnalisée. La multiplication des mouvements", de façon anarchique dans le tissu social et les défections de certains membres du gouvernement et du parti unique montrent l'autoritarisme d'un pouvoir présidentiel depuis l'indépendance ${ }^{12}$. C'est ainsi que de nombreux opposants tant de l'intérieur que les exilés ${ }^{13} \mathrm{n}$ 'ont pas manqué de donner du ton à la contestation sociale et politique dans la capitale ; la guerre et donc la fin du «havre de paix» donnent l'occasion à des opposants ${ }^{14}$ de s'exprimer dans le pays en organisant des manifestations multiformes. On peut donc affirmer que l'action militaire menée contre le pouvoir central a été «salutaire» pour une partie de l'opinion jusqu'à là étouffée par un régime politique mis en place en 1979. Mais cela démontre aussi la fin d'un mythe politique : la construction de l'unité nationale par un seul parti et de son fondateur. C'est aussi la rupture des relations de loyauté et de dépendance par rapport à un chef, un père de la Nation, Hassan Gouled Aptidon. La guerre civile et l'instabilité politique préoccupent la France.

\subsection{LA PRESSION FRANÇAISE SUR LE POUVOIR : NÉGOCIER AVEC LA RÉBELLION ET RÉFORMER LE RÉGIME POLITIQUE}

Située dans une position géostratégique, la République de Djibouti est une des pièces importantes du dispositif militaire français en Afrique, elle a la

\footnotetext{
11 Comme une compétition ouverte à tout le monde, sans règles, du jour au lendemain des groupuscules ethniques et claniques se créent et se lancent dans l'arène clano-politique. En fait, ces petits mouvements qui émergent en surface entre 1991 et 1993, existaient déjà dans le territoire et dans les pays limitrophes comme la Somalie. D'ailleurs, la nouvelle scène politique djiboutienne à partir de 1991 rappelle beaucoup celle de la Somalie où le clanisme politique s'exprime sur le terrain de la violence dans le vide du pouvoir central.
}

12 En effet le nouveau président de la République, élu par la Chambre des Députés (ancien nom du parlement monocaméral) adopte le 6 juillet 1977 un décret présidentiel par lequel il s'auto-attribue le titre de Chef de gouvernement. Acte qui a surprit la classe politique nationale, transformant le Premier ministre selon l'expression de André Laudouze, de «premier des ministres», il n'a pas de pouvoir constitutionnel comme son homologue français.

13 Le Chef de l'Etat niait l'existence d'opposants qui appelaient au boycott des élections législatives et présidentielles qui avaient lieu simultanément en avril 1987. Voir AFP, avril 1987.

14 Selon Jean Jacques Raynal, les courtisans de la veille se réveillent au matin opposants et face au vide du pouvoir, tout n'est pas aujourd'hui qu'opposition, op cit. 
première base à l'étranger.

C'est par une demande d'aide militaire que la France entre dans le jeu politique national à un moment de crise politique grave. Djibouti fait partie de ce pré carré qu'il faut gérer surtout dans cette période de troubles militaires, qui touchaient toute la Corne de l'Afrique pour une fois au même moment; l'instabilité politique de ce pays stratégique est plus dangereuse que celle d'un autre pays. Sa réaction était attendue tant par la jeune opposition que par le pouvoir, mais pas pour le même but. A un moment de changement politique dans les relations entre l'ex-métropole et les Etats postcoloniaux africains, la politique française était appelée à renouveler son discours et ses objectifs. Selon Marshall (1995) et Lamarche (1993) de nombreuses rencontres ont lieu entre responsables djiboutiens et conseillers français, elles n'aboutissent pas à un accord, parce qu'à la contradiction entre ces derniers s'ajoute l'opposition d'un courant conservateur du système politique djiboutien. Utilisant une culture franco-africaine basée sur des relations «patrimoniales» et personnelles, une rencontre au sommet entre François Mitterrand et Hassan Gouled ${ }^{15}$ est organisée à Paris, la crise entre les deux capitales prend officiellement fin. La France, qui a manifesté l'ouverture d'un dialogue entre les deux parties en conflit, a changé après cette rencontre. Si le dialogue fait partie toujours de la stratégie de la France dans la nouvelle situation politique djiboutienne, il n'est pas affirmé avec autant de fermeté. Mais la crise politique et la guerre civile lui offrent l'occasion de la réorienter sur le thème de la démocratisation en vogue à cette époque-là sans oublier le règlement du conflit armé entre les deux belligérants.

A l'issue de sa rencontre avec le président français, le dirigeant djiboutien s'engage à libéraliser son système politique à sa manière c'est-à-dire sans une intervention extérieure sur le modèle à suivre. C'est une concession qu'il a faite à la pression française, mais tout en sachant qu'il ne perd rien. Sans attendre un quelconque conseil de la part des conseilles français, il indique dans un discours de novembre 1991, soit un mois après le déclenchement des affrontements armés entre les deux parties au Nord, la voie qu'il a choisie pour opérer les changements nécessaires dans la vie politique en affirmant que : «à une date ultérieure, je consulterai le peuple djiboutien par la voie

15 Il faut rappeler qu'il a utilisé les relations «d'amitié politique» qui existaient entre les deux hommes dans les années 1960, quand Mitterrand était encore un opposant. 
La vie politique djiboutienne de 1992 a 2021 : quelques reflexions sur le changement et les reformes politiques

du référendum ou de la simple consultation populaire sur les changements à apporter dans le domaine politique, notamment par une représentation au sein des assemblées ainsi que la création d'organismes davantage rapprochées des populations ${ }^{16} .{ }_{.\rangle} \mathrm{Ce}$ dernier résume dans une formule lapidaire le sens de la guerre et des problèmes politiques qu'elle a créés par l'attribution des rebelles des postes au gouvernement ${ }^{17}$.

Le processus de changement politique commence donc par la violence. Mais le changement du discours politique djiboutien reflète un changement de la politique du côté des autorités djiboutiennes. Sachant qu'il va contrôler le processus de changement, «imposé» par une situation militaire et par la diplomatie française, il accepte de négocier avec les rebelles, mais pose des conditions dont l'un des points importants est l'absence de tout intermédiaire étranger entre les deux parties. Toutefois il y a eu des tentatives de rapprochement entre les deux parties par la diplomatie française, celle de l'Ethiopie et celle du Yémen.

Sur le terrain les affrontements armés continuent, mais cela n'empêche que la nécessité d'entreprendre un changement institutionnel et politique soit désormais acquise par les dirigeants politiques djiboutiens. A cet effet le pouvoir et l'opposition civile de l'intérieure se préparent à la mise en place d'un processus d'où l'importance de la méthode et de l'agenda des réformes pour le passage d'une situation à une autre.

\subsection{Méthode et agenda du Changement: la lutte par le contrôle du PROCESSUS ENTRE LES DIFFÉRENTES PARTIES}

Suivant l'analyse de Morlino, ici il s'agit «le comment» du changement en question. Cet auteur distingue le changement pacifique ou violent; dans le premier cas il y a des négociations entre les différents acteurs impliqués avec un ordre du jour et parfois sous la présidence d'une personnalité considérée neutre quant à la présidence et au déroulement des négociations. Autrement

\footnotetext{
$16 \quad$ La Nation du 27 novembre 1991.

17 Entretien du Président de la République dans Revue Le Courrier ACP-CEE, Nº174, mars-avril 1999. Ce dernier affirme que «pendant nos négociations, dit-il, avec le FRUD, nous avons compris qu'ils voulaient des postes au gouvernement.»
} 
dit une personne hors de la lutte politique comme les religieux ${ }^{18}$.

Après avoir reconnu la dégradation de la situation politique et le refus du soutien militaire français, le Chef de l'Etat réagit sur le plan politique. Une loi d'amnistie a été votée par l'Assemblée Nationale en début 1992, ce qui a permit le retour au pays des exilés politiques comme Aden Robleh Awaleh' ${ }^{19}$. C'est une mesure timide, mais importante qui marque le début de la libéralisation politique (O’Donnel, 1979), il y a une certaine ouverture du régime autoritaire par la reconnaissance de mouvements politiques et donc d'une opposition au régime.

Si cette loi est importante sur le plan politique, elle n'indique cependant pas la méthode à suivre pour introduire le changement politique tant attendu. Sur cette question, le président de la République a clarifié l'option qu'il allait suivre: la consultation de la population. Politiquement c'est une façon de marginaliser l'opposition naissante. Elle est menée par d'anciens ministres, comme feu Mohamed Djama Elabéro, Mohamed Ahmed Issa, Aden Robleh Awaleh" ${ }^{21}$, Ahmed Dini' ${ }^{22}$, qui dirigent des mouvements politiques, armés et/ ou civils. Ils proposent l'organisation d'une Conférence Nationale, ouverte

\footnotetext{
18 Dans les changements politiques qui ont eu lieu certains pays africains comme le Bénin, on a observé le rôle prépondérant joué par un religieux, l'archevêque de Cotonou a présidé la Conférence Nationale des Forces Vives de la Nationale. Le fait de présider cette conférence résulte d'un accord entre les différentes forces politiques et sociales du pays.
}

19 Aden Robleh Awaleh est une des personnalités politiques des années 1960, avocat de formation il fut Secrétaire Général du Front de Libération de la Côte Française des Somalis (FLCFS). Nommé ministre à différents postes après l'indépendance en 1977. Il fut le numéro 3 du Rassemblement Populaire pour le Progrès (RPP), un poste important. Mais sur décision du Président du parti, il est expulsé de l'unique formation politique du pays 1986, il sort du pays avec l'aide d'amis militaires. Arrivé en France, il critique le régime de parti unique, crée une organisation politique avec d'autres personnalités djiboutiennes.

20 C'est le président fondateur du Mouvement pour la Réconciliation et la Paix (MRP). Il a été ministre député depuis l'indépendance du pays. Il est considéré pendant un temps comme le candidat de la France pour succéder le Président Hassan Gouled Aptidon; il n’a été membre du comité exécutif du RPP.

21 Avocat de formation et leader indépendantiste dans les années 1960. C'est le président de l'Union des Mouvements Démocratiques (UMD), créé durant son exil en France.

22 Ancien ministre durant la période coloniale, dirigeant d'une formation politique afar. Critique contre le gouvernement de Mr. Ali Aref ; il s'allie avec Hassan Gouled Aptidon en vue de la formation d'un gouvernement d'unité nationale après l'indépendance. Rigoureux et fidèle à l'esprit de la constitution française du 4 octobre 1958 où il y a une répartition des pouvoirs constitutionnels entre les deux têtes de l'exécutif, il démissionne en décembre 1977, soit 6 mois après sa nomination comme premier Premier ministre de l'Etat postcolonial. Opposant au gouvernement, arrêté par la Police pour avoir créé un parti illégal en 1981. Libéré sur décision politique sans qu'il y ait procès. Depuis 1992 il est président du FRUD. 
La vie politique djiboutienne de 1992 a 2021 : quelques reflexions sur le changement et les reformes politiques

à la société civile comme le Bénin ou le Mali et un véritable bicéphalisme de l'exécutif23 à l'image du modèle français. Le fait de doter le $2^{\text {ème }}$ personnage du gouvernement des pouvoirs constitutionnels est pour l'opposition naissante une manière de redistribuer les ressources politiques et les prestiges qu'offre une place au sein de l'équipe gouvernementale. Le pouvoir rejette toutes ces propositions et choisit une autre voie: le recours aux travaux d'un groupe de personnalités. Le président de la République nomme une commission consultative constitutionnelle, chargée de lui faire des propositions de réformes, essentiellement constitutionnelles. Celle-ci est constituée en majorité par des proches du pouvoir, les leaders de l'opposition contestent sa composition. L'origine professionnelle de ses membres est variée; on trouve des représentants politiques, des hommes d'affaires ${ }^{24}$, du monde religieux ${ }^{25}$. Elle est présidée par le Président de l'Assemblée Nationale. Les séances de travail de la commission sont marquées par des fortes divergences entre les participants. L'avant-projet ne fait pas l'unanimité des membres de ladite commission. Mais sa constitution et son objectif montrent l'attitude d'un pouvoir fort ou qui se renforce ; il impose des réformes et cherche par là à contrôler férocement l'évolution politique et sociale du pays. Le rapport de ses travaux est adressé au Président de la République, qui tire personnellement une conclusion. Il est le seul à décider de la suite à donner à un tel texte. Dans une telle configuration politique, il va de soit que le principal objectif du pouvoir en place est le contrôle du processus engagé. Donc le changement institutionnel est marqué par l'attitude d'un gouvernement qui ne fait pas de place au consensus tant

\footnotetext{
23 Les opposants proposent un Premier ministre, qui serait le Chef du gouvernement, responsable devant l'assemblée nationale, donc un partage du pouvoir au sommet de l'Etat. En fait, c'est une reproduction du modèle français de la $5^{\text {me }}$ République. Cette demande est une stratégie politique de ces derniers qui espéraient un Premier Ministre nommé dans ses rangs en cas de réponse favorable à leur demande et de victoire électorale. Un tel cas de figure s'est produit dans d'autres pays africains à la même époque.
}

24 Il faut noter la présence de l'ex président de la Chambre de Commerce et d'Industrie de Djibouti, c'est un des acteurs politiques des années 1960 et 1970 ; il contrôle une part importante des activités industrielles locales. Alain Renaud XX

25 S'agissant des milieux d'affaires et religieux, les relations que le pouvoir entretient avec les représentants de ces milieux sont tellement favorables, parce que le pouvoir autoritaire est un tout, donc totalitaire. L'élite économique n'est pas indépendante de l'élite politique, si elles ne se confondent pas, le contrôle de l'une sur l'autre et le soutien mutuel des membres favorisent une certaine identité des intérêts. La participation de l'élite économique à la commission est une manifestation concrète des relations étroites qui existent entre hommes d'affaires et hommes politiques. Ce n'est pas un fait propre aux systèmes sociaux africains; on n'en trouve ailleurs dans le monde. Il faut noter l'absence de tout représentant de la société. 
África (São Paulo, 1978, Online), São Paulo, n. 42, p. 177-219, 2021

avec des responsables du mouvement social que les opposants politiques. Le Chef de l'Etat s'adresse à la population par un discours radio-diffusé le 27 juin 1992, jour de l'indépendance du pays. Le principal point politique de cette intervention est la convocation de l'électorat pour un référendum le 4 septembre 1992 sur l'adoption d'une nouvelle constitution et du multipartisme. L'opposition est divisée, certains leaders appellent sans enthousiasme à voter «oui» au référendum tandis que le FRUD a lancé un appel au boycott. Deux questions sont soumises aux électeurs:

$-1^{\circ}$ Approuvez-vous le projet constitutionnel ${ }^{26}$ ?

- $2^{\circ}$ Approuvez-vous le multipartisme limité à quatre partis?

L'adoption d'une constitution libérale et la reconnaissance du multipartisme sont, selon Morlino, un changement qui affecte à deux aspects importants du régime politique: le fondement juridique, la constitution et la participation politique, c'est-à-dire la représentation des différentes forces politiques et sociales au sein des institutions politiques. Donc en partant de la situation antérieure, sur ce point il y a un changement d'une part par la reconnaissance du libéralisme politique, l'expression de différentes opinions politiques et par conséquent la reconnaissance du droit à la participation de la vie politique de la cité par tous les citoyens sans discrimination.

Seuls les habitants des régions non touchées par la guerre, la capitale, sa région et une ville du Sud-Ouest, Ali-Sabieh, la capitale et sa région, et quelques citoyens vivant à l'étranger ont pu voter à ce référendum. Les habitants des villes du Nord, Tadjourah et Obock et les villages, situés à la frontière, se sont réfugiés ${ }^{27}$ en Ethiopie. Selon les chiffres officiels publiés par le ministère de l'Intérieur, qui ne prennent en compte qu'une partie des citoyens, $96,84 \%$ des électeurs se sont en faveur de la première question, qui est aussi la plus importante des deux points du référendum. Quant à la deuxième,

26 Le projet constitutionnel soumis au peuple est une constitution libérale, qui diffère sur beaucoup de point aux Lois Constitutionnelles, votées progressivement entre juin 1977 à février 1981. En effet dans le nouveau texte, des libertés fondamentales sont reconnues aux citoyens, le titre II y exclusivement est consacré. Mais la constitution ne se limite pas seulement à une reconnaissance théorique de ces libertés, il est prévu des institutions et des mécanismes qui les garantissent comme par exemple le conseil constitutionnel et surtout l'art.80 de la constitution qui prévoit la possibilité pour un justiciable de soulever la question de l'inconstitutionnalité d'un texte de loi lors d'un procès; lequel est suspendu et c'est la Cour Suprême qui décide s'il y a lieu de consulter le conseil constitutionnel.

27 Et au moment où avait lieu cette consultation, ils étaient encore en territoire éthiopien, puisque la guerre n'était pas finie, ce qui réduit la portée politique qu'une telle consultation pourrait avoir. 
La vie politique djiboutienne de 1992 a 2021 : quelques reflexions sur le changement et les reformes politiques

$96,79 \%$ de l'électorat a voté pour ${ }^{28}$.

Mais dans le contexte sociopolitique de 1991 et 1992 - les affrontements militaires continuent toujours dans le Nord et le Sud-Ouest du pays l'organisation d'une consultation sur le devenir constitutionnel du pays est une opération politique détournée; elle répond à un calcul politique d'un noyau dur des dirigeants proches du président, pressés pour se maintenir au pouvoir. Il faut savoir que tous les partisans du parti unique et donc du système en place ne peuvent tous tirer profit de la nouvelle «donne politique». Dès lors, les résultats ne peuvent surprendre personne, et la présence d'observateurs étrangers ${ }^{29}$ ne change en rien le déroulement du processus engagé, du programme politique du pouvoir et la stratégie mise en place par ceux qui contrôlent l'appareil étatique et administratif. La nouvelle constitution maintient la même forme du gouvernement, c'est-à-dire un pouvoir exécutif bicéphal $^{30}$ et un parlement monocaméral (l'Assemblée Nationale), elle n'instaure pas un partage des pouvoirs entre les deux têtes de l'exécutif. Elle renforce les pouvoirs du président de la République, celui-ci partage l'initiative parlementaire avec les députés (art.59 de la constitution).

A la suite des résultats du référendum, considérés comme un aval politique donné par l'électorat au pouvoir en place, un autre processus se déclenche: le développement d'un cadre juridique conforme à la constitution libérale ainsi

28 Sans révision des listes électorales depuis les dernières élections de 1987, on doit prendre les chiffres publiés sur la participation et les réponses avec la prudence qui s'impose dans l'interprétation à faire sur le plan sociopolitique dans un contexte où il n'y a pas de dialogue entre les forces politiques.

29 Ces observateurs, qualifiés par certains opposants comme de pèlerins électoraux, sont en fait dans leur majorité des parlementaires nationaux ou du parlement européen, il y a aussi des représentants d'organisations gouvernementales comme l'OUA, La Ligue Arabe...Ils sont invités par le pouvoir en place le plus souvent à la suite d'une demande insistante de l'opposition, qui a peur des fraudes et autres malversations durant le jour de l'élection. Ces derniers n'ont ni les moyens, ni la faculté de vérifier les opérations qui précèdent l'acte de voter, de mettre un papier dans une urne. Ils répondent juste, à une demande de leur parlement d'origine ou de leur organisation. L'observation des élections nationales, générales le plus souvent est une question très sensible pour un gouvernement et c'est ce qui explique en partie la limitation du rôle de ces derniers en Afrique ou ailleurs dans le Tiers-Monde.

30 L'exécutif djiboutien est un exécutif bicéphal «déséquilibré» à faveur du premier personnage de l'Etat, c'est-à-dire le Président de la République, qui cumule le statut de Chef du gouvernement. La figure de Premier ministre n'existe que pour une raison de répartition ethnique du pouvoir politique dans le nouvel Etat postcolonial. Il n'est pas le chef de file du parti gagnant des élections législatives. Sa nomination dépend d'une volonté personnelle du seul électeur à ce poste : le chef de l'Etat. Ce genre d'exécutif bicéphal avec une forte prépondérance du président de la République on le trouve aussi dans d'autres pays africains, au Cameroun, en Côte d'Ivoire, au Gabon, Sénégal... 
África (São Paulo, 1978, Online), São Paulo, n. 42, p. 177-219, 2021

votée. L'assemblée Nationale vote deux lois organiques, la première relative aux élections et la seconde relative aux partis politiques. La première institue, et c'est une innovation institutionnelle, une commission de supervision des élections. $\mathrm{Ce}$ n'est pas une institution indépendante de l'administration et du gouvernement, elle ne donne qu'un avis au Président de la République qui décide la date de la convocation de l'électorat, le nombre et l'implantation des bureaux de vote ${ }^{31}$ par décret présidentiel. Ce sont des questions importantes dans une compétition électorale. Quant à la deuxième loi, elle place le ministère de l'Intérieur au centre de la procédure de reconnaissance légale des partis politiques. Mais ce qui est important à noter, c'est la définition du parti politique. En fait le parti politique n'est pas définit par cette loi non plus par la constitution ${ }^{32}$ Aussitôt après la publication des résultats du référendum, certains dirigeants de l'opposition déposent des demandes de reconnaissance légale au ministère de l'Intérieur. Le nombre des partis politiques est limité à quatre pour une décennie. C'est donc un multipartisme limité qui est instauré. Le nombre de partis n'est pas significatif, par contre ce importe c'est l'attitude du pouvoir en place et de son administration à propos de la légalisation donc de la reconnaissance aux mouvements qui existent comme des partis politiques ayant le droit de présenter des candidats aux différentes élections. Ledit ministère serait-il libéral ou plus restrictif? Quels seraient ses choix dans la procédure de légalisation? C'est sans problème que le mouvement, créé par l'ancien ministre de la Santé et des Affaires Sociales, Mohamed Djama Elabé, est légalisé sous la dénomination de Parti du Renouveau Démocratique (PRD). Alors que celui d'un ancien ministre, Aden Robleh Awaleh, a eu quelques difficultés pour l'obtention de sa reconnaissance légale, il a été finalement légalisé sous la dénomination de Parti National Djiboutien (PND). D'autres demandes ont été rejetées alors que la limite constitutionnelle de quatre partis n'a été atteinte. Ce refus peut s'expliquer par une décision du pouvoir d'exercer un contrôle sur l'institutionnalisation des formations poli-

31 L'article. 41 de la Loi Organique $\mathrm{N}^{\circ}$ 1/AN/92 relative aux élections prévoit clairement la compétence du gouvernement dans le nombre et l'implantation des bureaux de vote, cet article dispose que: «Après avis des commissions de supervision des élections, le président de la République, sur demande du Ministre de l'Intérieur, fixe par arrêté le nombre et l'implantation des bureaux de vote et en désigne les membres.»

32 C'est dans le titre II de la constitution et spécialement l'art. 15 qu'on peut trouver une définition de la constitution. Il est reconnu aux citoyens ont le droit de constituer des associations, et en considérant la forme de la participation politique et sociale prévue par le nouveau cadre constitutionnel, le parti politique est donc une association à but non lucratif, mais dont l'objet est différent de celui des autres. 
La vie politique djiboutienne de 1992 a 2021 : quelques reflexions sur le changement et les reformes politiques

tiques, donc de l'opposition. D'autres analyses mettent l'accent le fait ethnique. En effet, les leaders des nouveaux partis sont tous du même groupe ethnique, même si leur composition est multiethnique en application de la loi sur les partis politiques votés en octobre 1992, qui exclut la constitution d'une formation à base ethnique. En effet la légalisation des mouvements en partis politiques donc en acteurs officiels de la nouvelle scène politique djiboutienne est bien contrôlée ; certains leaders pour des raisons diverses et particulièrement de leur passé ont «facilement» le certificat du ministère de l'Intérieur tandis que d'autres se voient opposés un refus. Il y au total trois formations politiques en incluant le parti au pouvoir, qui ne s'est pas affecté par la législation sur les partis politiques.

L'adoption d'une constitution reconnaissant le pluralisme politique est une étape importante pour l'instauration d'un nouveau régime politique en République de Djibouti, son existence n'est que symbolique si une nouvelle culture n'accompagne pas.

L'analyse du processus de changement conduit à la chercheuse ou au chercheur à prendre en considération d'autres éléments, et l'un d'eux est l'organisation d'élections pluralistes, libres et compétitives. D'où l'importance d'un sous-processus : le système électoral. Cependant dans un contexte comme celui de la République de Djibouti des années 1990, l'étude d'un tel système consiste à démontrer non seulement le changement de la représentation tant sociale que politique, mais aussi de ses conséquences politiques pour les entrepreneurs politiques, que ça soit au niveau individuel que collectif.

\section{LA COMPÉTITION ÉLECTORALE PLURALISTE : FONDEMENT D'UN NOUVEAU RÉGIME POLITIQUE}

Sur le plan politique la mise en pratique du pluralisme se fait par l'intermédiaire de l'organisation d'élections libres et ouvertes à tous les acteurs politiques remplissant les conditions posées par la législation électorale. Plus qu'une simple technique de choix des élus nationaux et locaux, les élections pluralistes constituent théoriquement un enjeu important pour l'institutionnalisation $^{33}$ de la conquête du pouvoir politique dans le nouveau régime politique.

\footnotetext{
33 Quand on parle d'institutionnalisation on fait souvent référence aux régimes militaires, qui s'installent à la suite d'un coup d'Etat. Or la pérennisation au pouvoir d'un homme politique civil - le cas de ce qu'on a appelé "les Pères de la Nation" ou leurs dauphins désignés constitue aussi un handicap pour le progrès poli-
} 
África (São Paulo, 1978, Online), São Paulo, n. 42, p. 177-219, 2021

\subsection{L'ENJEU DES ÉLECTIONS: ALTERNANCE ET INSTITUTIONNALISATION PACIFIQUE DE LA PRISE DU POUVOIR}

Dans un régime pluraliste, l'élection représente un élément central dans le jeu politique national et local.

Dans la constitution djiboutienne du 4 septembre 1992, l'élection au suffrage universel est le mode retenu pour les fonctions politiques comme la présidence de la République ${ }^{34}$, rééligible qu'une seule fois, et la députation ${ }^{35}$ pour un mandat de 5 ans à l'Assemblée Nationale tout comme dans les municipaux et conseils régionaux ${ }^{36}$. Or ce n'est pas une nouveauté en soi parce que sous l'ancien régime les dirigeants étaient aussi élus, il y avait une liste unique. La nouveauté, c'est la nature de l'élection ${ }^{37}$. Elle est théoriquement compétitive dans la mesure où plusieurs candidats, présentés par différentes formations politiques, se présentent aux postes à pourvoir. Mais au-delà d'un choix offert aux citoyennes/s entre les programmes des entrepreneurs politiques, la compétition électorale a une autre fonction : l'institutionnalisation pacifique de la prise du pouvoir dans un régime démocratique.

Les premières élections législatives dites pluralistes, «démocratiques» et «libres» dans l'histoire politique de la République de Djibouti ont lieu le 18 décembre 1992, soit trois mois après le référendum constitutionnel ${ }^{38}$. Prévues

tique de la société. Mais dans un cas comme dans l'autre, l'institutionnalisation permet une certaine alternance pacifique quant à la prise du pouvoir supprime de l'Etat.

34 L'article 23 dispose que : «Le Président de la République est élu pour six ans au suffrage universel direct et au scrutin majoritaire à deux tours. Il n'est rééligible qu'une seule fois.»

35 L'article 46 al 1 dispose que : «Les députés à l'Assemblée nationale sont élus pour cinq ans au suffrage universel direct et secret. Ils sont rééligibles».

36 La constitution du 4 septembre 1992 reconnaît dans les articles 85 et 86 l'existence de collectivités territoriales. Contrairement à d'autres domaines de la vie institutionnelle et politique où des lois organiques ont été adoptées entre 1992 et 1994, la loi sur la décentralisation a été adoptée en juillet 2002 juste après le deuxième accord de paix avec la rébellion afar en mai 2000. Or un an avant cette date, des conseils régionaux sont mis en place, ils sont présidés par les Commissaires de la République, Chef lieu du district, des okal et autres acteurs de la société civile naissante y participent. Mais ils n'ont encore pas réellement une autonomie comme prévoit la constitution.

37 La participation aux élections n'est plus le fait d'un parti unique institutionnalisé, elle n'est plus un plébiscite qui renforce un pouvoir autoritaire. Aussi, si les partis continuent de présenter des candidats, pour les élections présidentielles, la loi organique sur les élections votée en 1992 permet à des candidats indépendants de se présenter.

38 Elles ont été Le statut de parti appelle à des changements non seulement de structure, mais aussi au 
La vie politique djiboutienne de 1992 a 2021 : quelques reflexions sur le changement et les reformes politiques

le 20 novembre 1992 ; elles ont été remportées à la demande de nouvelles formations politiques légalisées, lesquelles n'ont pas eu le temps de se préparer sur le plan politique et sur le plan financier. Jusqu'à l'obtention de la reconnaissance légale comme parti politique, ils étaient des mouvements politiques non structurés. En ce temps record d'organisation des élections fondatrices du nouveau système politique, en réalité le parti au pouvoir cherchait une nouvelle légitimité politique dans le nouveau contexte politique. Les partis récemment légalisés, émettent des réserves. En fait, l'opposition sur la date retenue occulte une autre opposition, plus fondamentale, celle qui porte l'accès et le contrôle des ressources publiques, qui déterminent plus ou moins les résultats électoraux. Et l'une de ces ressources, c'est le fichier électoral, contrôlé par le gouvernement et la distribution des cartes d'électeurs.es.

Résultats des élections législatives du 18 décembre 1992

\begin{tabular}{|l|l|l|}
\hline \multicolumn{1}{|c|}{ Coalitions et partis } & Suffrages & \multicolumn{1}{|c|}{ Nombre de sièges } \\
\hline $\begin{array}{l}\text { Rassemblement Populaire pour le } \\
\text { Progrès (RPP) }\end{array}$ & $65 \%$ & 65 \\
\hline $\begin{array}{l}\text { Parti du Renouveau Démocratique } \\
\text { (PRD) }\end{array}$ & $25 \%$ & 0 \\
\hline
\end{tabular}

Sources : Ministère de l'Intérieur.

Depuis la libéralisation politique à la fin de 1991, l'opposition djiboutienne est composée de multiples mouvements politico-claniques; elle tente de s'organiser politiquement autour d'une structure : Union de l'Opposition Djiboutienne $(\mathrm{ODU})$, qui sert de forum de rencontre et de rapprochement des dirigeants de ces mouvements sur le processus de changement politique engagé. A l'issue d'une réunion à Paris le 18 juin 1992, leurs leaders ont convenu de boycotter les élections législatives. Mais le boycot n'est pas suivi de la même manière par les deux nouveaux partis de l'opposition; le PRD présente des candidats et se démarque ainsi de l'accord signé à Paris par les mouvements de l'opposition. Sa participation a fait l'objet d'un vote au comité central du parti, 7 membres sur 11 se sont prononcés en faveur. Le troisième parti, le PND, n'a pas présenté des candidats et a justifié son refus d'y prendre part par le contrôle du parti gouvernemental sur le processus administratif et 
África (São Paulo, 1978, Online), São Paulo, n. 42, p. 177-219, 2021

législatif comme la délimitation des circonscriptions électorales et l'absence de révision des cartes d'électrices/eurs. Ce sont des questions, qui selon son leader Aden Robleh Awaleh, n'ont pas fait l'objet d'un consensus entre les formations politiques. Il a dénoncé le scrutin retenu pour cette consultation : the winner takes all ${ }^{39}$. Selon les chiffres publiés par le ministère de l'Intérieur, le parti gouvernemental a remporté ces élections avec $74,59 \%$ des suffrages exprimés contre $25,40 \%$ pour le $\mathrm{PRD}^{40}$, tous les sièges du parlement sont occupés par la coalition RPP-FRUD ${ }^{4}$. Les deuxièmes élections législatives ont lieu en décembre 1997.

Résultats des élections législatives du 18 décembre 1997

\begin{tabular}{|l|l|l|}
\hline \multicolumn{1}{|c|}{ Coalitions et partis } & \multicolumn{1}{c|}{ Suffrages } & \multicolumn{1}{c|}{ Nombre de sièges } \\
\hline Coalition RPP-FRUD & $74,59 \%$ & 65 \\
\hline PRD & $25,40 \%$ & 0 \\
\hline
\end{tabular}

Sources: Ministère de l'Intérieur.

Par rapport aux premières élections législatives pluralistes, il faut noter un changement d'opinion des leaders de l'opposition civile ; les quatre partis y prennent part. Cependant il y a seulement trois listes: celles de deux partis de l'opposition et celle de la coalition gouvernementale RPP-Frud ${ }^{42}$. Compte tenu du contexte sociopolitique, c'est sans surprise que cette dernière remporte ces élections grâce au contrôle de l'appareil administratif, l'absence de

39 Le scrutin majoritaire à deux tours, il ne reflète pas l'expression de l'électorat national. En effet une partie de l'électorat, qui a voté pour le seul parti de l'opposition n'est pas politiquement représentée.

40 Par stratégie électorale ou par les difficultés financières qu' affrontent les nouvelles formations politiques, le PRD n'a présenté des candidats que dans 3 districts sur 5 : la capitale, Djibouti-ville, Ali-Sabieh et Dikhil. Le leader du parti est originaire du District de Dikhil, et donc il avait un espoir de remporter quelques sièges des 12 sièges attribués par la loi à ce district.

${ }_{41}$ Alors que les dirigeants du parti gouvernemental se sentent investis d'une «légitimité populaire», son rival le RPP Le PRD a contesté les résultats. Au lieu d'appeler à la violence, il a plutôt opté la voie pacifique en déposant des requêtes pour annulation à la Cour Suprême pour les élections législatives de 1992. Le conseil constitutionnel n'étant pas encore fonctionnel.

42 De même qu'en 1992, les deux partis de l'opposition adoptent une stratégie de concentration de leur chance à des districts où l'électorat leur paraît favorable surtout le plan ethnique. Ainsi le PND - qui participe pour la première fois - présente des candidats seulement dans le district d'Ali Sabieh, où est originaire son président; donc il n'avait qu'une possibilité limitée d'avoir des élus, alors que le PND présente ses candidats dans trois districts. Mais quelle que soit la stratégie adoptée, s'il ne remporte pas plus de la moitié des 37 sièges de la capitale, la victoire dans les autres districts est insignifiante. 
La vie politique djiboutienne de 1992 a 2021 : quelques reflexions sur le changement et les reformes politiques

réforme des listes électorales et surtout à l'effet d'un système électoral inique. En effet le parti qui remporte la totalité des sièges de la capitale (37 sur 65) est déclaré vainqueur, ce qui exclut de facto l'organisation d'un deuxième tour, prévu par la législation électorale. C'est la première fois que le parlement djiboutien accueille des députés d'un autre parti que ceux de l'ex parti unique. Les élections législatives pluralistes de 1992 et de 1997 ne sont pas tellement compétitives, auncun député de l'opposition n'est élu.

Au terme de 10 ans d'un multipartisme limité à 4 formations ${ }^{43}$, le multipartisme intégral est instauré en 2002. Ainsi des nouvelles formations politiques voient le jour, dirigées par d'anciens ministres et de hauts fonctionnaires de l'Etat, responsables du RPP comme Ahmed Dini Ahmed, ancien Premier ministre et ancien président du FRUD, Moumin Bahdon Farah, ancien Secrétaire Général du RPP et ministre des Affaires Etrangères et ministre de la Justice et des Affaires musulmanes et Ismaël Guedi Hared, ancien Directeur de Cabinet du président Hassan Gouled Aptidon. Le mandat des députés est de 5 ans. Les troisièmes élections du nouveau régime n'ont pas lieu en 2002 comme prévu, sans doute pour des raisons financières quant à leur organisation, elles ont lieu en janvier 2003, opposant une nouvelle coalition de 4 partis dont l'ex parti unique RPP, appelée Union pour la Majorité Présidentielle (UMP) et une coalition 4 partis de l'opposition Union de l'Alliance pour la Démocratie (UAD). Selon les résultats officiels publiés par le ministère de l'Intérieur, la coalition UMP remporte la totalité des sièges. La victoire de cette coalition se répète aux quatrièmes et cinquièmes élections législatives respectivement en 2008 et 2013 . Et à chaque fois, l'opposition dépose des recours, lesquels sont rejetés toujours par le Conseil Constitutionnel ${ }^{44}$.

Mais ce n'est qu'à la suite de l'introduction du système électoral proportionnel en novembre 2012, que l'opposition a fait son entrée dans le parlement. Il y a une certaine régularité des élections législatives, même si la fin du

\footnotetext{
43 Ces nouvelles formations politiques sont créées par d'anciens ministres, qui ont démissionné ou été exclus de l'ex parti unique et condamnés 5 ans d'interdiction d'exercice de leurs droits politiques et de nouvelles personnes du paysage politique national.

44 Mohamed Abdillahi Bahdon, «La jurisprudence du Conseil Constitutionnel de la République de Djibouti en matière électorale», Revue de Droit Africain n⿳31, juillet 2004, pp. 417-423. Et Mohamed Abdillahi Bahdon, «La juridiction constitutionnelle en République de Djibouti : enjeux et perspectives de la garantie du respect des droits et des principes fondamentaux.», Cuestiones Constitucionales, Julio-Diciembre de 2006, p.3-32.
} 
mandat n'est pas respectée comme en 2003. Au lieu d'organiser en 2002, le gouvernement reporte de quelques mois le mandat de la législature 19922002. Ce n'est en janvier 2003 qu'elles ont lieu.

Si les résultats des élections législatives post réforme constitutionnelle n'ont pas varié, celles du 22 février 2013 ont marqué un tournant dans la vie politique djiboutienne. Effet de la proportionnelle introduite en 2012 ou une décision du gouvernement de respecter la volonté de l'électorat ou une meilleure mobilisation de la coalition de l'opposition, celle-ci se proclame vainqueure.

Résultats des élections législatives du 22 février 2013

\begin{tabular}{|l|c|c|}
\hline \multicolumn{1}{|c|}{ Coalitions et partis } & Suffrages & Nombre de sièges \\
\hline Union pour la Majorité Présidentelle (UMP) & $49,39 \%$ & 55 \\
\hline Union pour le Salut National (USN) & $47,61 \%$ & 10 \\
\hline Centre des Démocrates Unifiés (UDC) & $3 \%$ & 0 \\
\hline
\end{tabular}

Source: ministère de l'Intérieur. Elaboration personnelle.

Les élections du 23 février 2018 n'ont pas dérogé à la règle, la victoire écrasante de la coalition au pouvoir. Cependant par rapport aux élections précédentes, des partis de l'opposition ont boycotté ces élections comme le Mouvement pour le Renouveau Démocratique (MRD), le Parti National Démocratique (PND), Mouvement pour le développement (ou la démocratie) et la liberté (Model), Rassemblement pour l'action de développement et la démocratie (RADD). Mais c'est la deuxième fois que l'opposition fait son entrée au Parlement et une présence de la femme, des 65 députés.es, 15 sont des femmes.

Résultats des élections législatives du 23 février 2018

\begin{tabular}{|l|l|l|}
\hline \multicolumn{1}{|c|}{ Coalitions et partis } & \multicolumn{1}{|c|}{ Suffrages } & Nombre de sièges \\
\hline $\begin{array}{l}\text { Union pour la Majorité Présidentelle } \\
\text { (UMP) }\end{array}$ & $87,83 \%$ & 57 \\
\hline $\begin{array}{l}\text { Coalition Union pour la Démocratie et la } \\
\text { Justice (UDJ) et Parti Djiboutien pour le } \\
\text { Développement (PDD) }\end{array}$ & 10,92 & 7 \\
\hline Centre des Démocrates Unifiés (UDC) & $0,68 \%$ & 1 \\
\hline $\begin{array}{l}\text { Alliance Républicaine pour le } \\
\text { Développement (ARD) }\end{array}$ & 0,57 & 0 \\
\hline
\end{tabular}


La vie politique djiboutienne de 1992 a 2021 : quelques reflexions sur le changement et les reformes politiques

Source: ministère de l'Intérieur. Elaboration personnelle.

A première vue le principal avantage de ces coalitions est le fait pour les deux coalitions de présenter des candidats dans tous les districts, parce qu'elles disposent les moyens financiers. Or on a constaté que la coalition de l'opposition n'a pas présenté des candidats.es dans toutes les régions. Est-ce pour une raison financière ou de stratégie ? En effet, pour l'opposition, c'est une question de stratégie de présenter des candidats.es dans les régions où elle pourrait gagner contre sa concurrente. L'autre avantage de la constitution de coalition, c'est l'idée de gestion des affaires de l'Etat, non par un seul parti, sinon un groupe de formations, ce qui introduit le fait d'avoir des négociations quant à la répartition des ministères, la mise en place des nouvelles institutions et de nouvelles politiques.

Même s'il y a la routine, c'est-à-dire la victoire de la coalition au pouvoir, toutefois chaque élection législative est particulière et différente par rapport aux précédentes tant le contexte politique change, des nouvelles formations politiques se présentent. Sans consensus avec les partis de l'opposition, le gouvernement reforme la loi électorale ${ }^{45}$ de 1992. Mais la lutte continue entre un pouvoir dont le principal objectif est de se maintenir et l'opposition qui tente de gagner du terrain pour diriger le pays.

Depuis la législature 2003-2008, le Parlement n'est plus unisexe. La femme a fait son entrée. Plus qu'un combat des femmes, c'est le système de quota les a permis d'être élues députées; la majorité d'entre elles sont de la coalition au pouvoir.

Présence de la femme djiboutienne au Parlement

\begin{tabular}{|c|c|}
\hline Législature & Nombre d'élues \\
\hline $2003-2008$ & $\mathbf{7}$ \\
\hline $2008-2013$ & $\mathbf{9}$ \\
\hline $2013-2018$ & $\mathbf{7}$ \\
\hline $2018-2023$ & $\mathbf{1 5}$ \\
\hline
\end{tabular}

Source: ministère de l'Intérieur. Elaboration personnelle.

L'augmentation du nombre de femmes élues dans l'actuelle législature est l'effet d'une nouvelle loi sur le quota. Le gouvernement modifie la loi

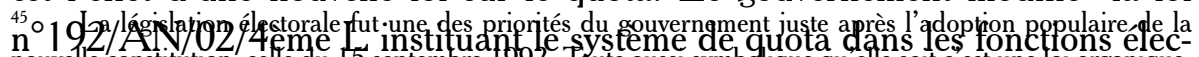
nouvelle constitution, celle du 75 septembre 592 . Soute aussis symbollique qu elle solt c est une lor organique.

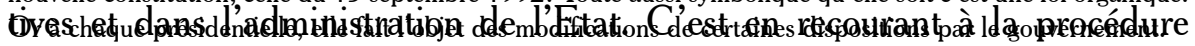


África (São Paulo, 1978, Online), São Paulo, n. 42, p. 177-219, 2021

d'urgence que la nouvelle loi est publiée. Elle est adoptée en janvier 2018. Son article stipule que "Le quota des femmes élues à l'Assemblée Nationale est fixé au moins à 25\%". Ainsi le quota des femmes à l'Assemblée Nationale passe de $10 \%$ à $25 \%$. Said Ibrahim Badoul, ex le président de l'Assemblée Nationale, déclarait que «l'arrivée des femmes insufflera une nouvelle vie au parlement, institution jusque-là exclusivement réservée aux hommes ${ }^{46 \%}$. La première candidate est présentée par le $\mathrm{PRD}$, avant l'adoption du système de quota, en 1997. D'autres pays africains ont atteint 30\% le quota comme l'Afrique du Sud, le Mozambique Mais l'entrée des femmes au parlement est être symbolique. L'activité parlementaire se fait dans les commissions et le bureau de la chambre parlementaire. Des huit commissions parlementaires, seule une était présidée par une députée dans la législature 2013-2018. Aucune députée n'a occupé les postes de vice-présidence et le secrétariat des commissions.

Quant aux élections présidentielles à multiple candidature ${ }^{47}$, les premières ont lieu le 7 mai 1993 dans un contexte sociopolitique difficile. L'enjeu de celles-ci est tellement important compte du rôle central que joue le président $^{48}$ de la République dans la vie politique nationale. Malgré les conditions financières ${ }^{49}$ pour se présenter à la présidence de la République, elle oppose 5 candidats dont le chef de l'Etat et deux indépendants $\mathrm{s}^{50}$. Deux sont

46 Une journée de sensibilisation des femmes à la vie politique ADI 18/12/2002, www.adi.dj

47 Il faut noter la différence que pose la législation électorale entre les élections législatives et les élections présidentielles. Aux termes de l'art.10 du décret 92-0125/PR/MI fixant les modalités d'organisation du scrutin du 18 décembre 1992, seuls les partis politiques légaux sont habilités à présenter des candidats aux élections législative. Par contre à propos des élections présidentielles, la loi ne précise rien quant à la candidature indépendante, mais elle ne pose pas non plus une interdiction de ce type de candidature.

48 La constitution votée le 4 septembre 1992 n'a pas modifié substantiellement les pouvoirs du Président de la République, au contraire elle les a renforcés avec l'initiative parlementaire, qu'il partage avec les députés. Il est toujours Chef du gouvernement, chef des armées, président du Conseil Supérieur de la Magistrature, président du parti gouvernemental et garant des institutions publiques.

49 L'art. 23 de la Loi Organique N ${ }^{\circ} 1 / \mathrm{AN} / 92$ du 29 octobre 1992 dispose que les candidats doivent déposer une caution de 5. 000. 000 Franco Djibouti au Trésor Public. Le montant est très élevé pour les nouveaux acteurs politiques, ce qui les dissuade à participer à la compétition électorale. La loi électorale prévoit le remboursement de la caution quand le candidat a obtenu plus de 10\% des suffrages exprimés. (art. 28). Les 5 candidats ont occupé des hautes responsabilités politiques et gouvernementales dans les années 1970 et 1980 ; deux ont été ministres sous Hasse Gouled Aptidon : Mohamed Djama Elabé (PRD) et Aden Robleh Awaleh (PND), un diplomate et un ex conseiller à la présidence.

50 Deux des cinq candidats n'ont pas l'appui financier et d'organisation matérielle d'une formation poli- 
La vie politique djiboutienne de 1992 a 2021 : quelques reflexions sur le changement et les reformes politiques

donnés favoris, le président sortant Hassan Gouled Aptidon et le leader du Parti du Renouveau Démocratique, Mohamed Djama Elabé.

Elections présidentielles du 7 mai 1993

\begin{tabular}{|l|l|}
\hline \multicolumn{1}{|c|}{ Candidats } & Pourcentage des votes \\
\hline Aden Robleh Awaleh & $12,99 \%$ \\
\hline Hassan Gouled Aptidon & $60,76 \%$ \\
\hline Mohamed Djama Elabe & $22,03 \%$ \\
\hline
\end{tabular}

Sources: ministère de l'Intérieur. Elaboration personnelle.

Plus de $80 \%$ des suffrages sont répartis entre ces trois candidats, les deux autres se partagent un plus de $3 \%$ des suffrages exprimés. Le taux de participation est estimé à $48 \%$ du corps électoral. La proclamation des résultats n'a pas dégénéré des actes violents ou des dans les villes; deux des candidats de l'opposition ont déposé des recours pour fraudes et annulation au conseil constitutionnel ${ }^{51}$. L'une des nouveautés institutionnelles introduites est l'institutionnalisation d'une justice constitutionnelle dont l'une des attributions est la résolution des conflits liés aux résultats des élections. Dans les deux cas, les requêtes ont été rejetées par les organes judiciaire et juridictionnel.

«Fort» d'une légitimité électorale dans un contexte sociopolitique difficile, le président Hassan Gouled appelle à l'opposition armée de déposer les armes et de négocier avec lui et son gouvernement. Pour lui, le FRUD n'a plus d'excuses pour continuer la guerre, car avec le référendum populaire sur la nouvelle constitution et les élections de décembre 1992 et de mai 1993, la démocratie est «instaurée», répondant ainsi à un des points du programme politique du FRUD ${ }^{52}$. Or par manque des moyens financiers et matériels et/ou par politiques personnelles - division interne, la rébellion se divise en deux parties à la fin de l'année 1993. Cette division est pu-

tique pour leurs compagnes électorales, Moussa Ali Tourtour, ancien conseiller économique du Président de la République et Ahmed Abdi, ancien diplomate.

51 Le conseil constitutionnel n'est pas une institution nouvelle; elle a existé sous la période de parti unique, mais n'a jamais exercé ses fonctions. Le monopole de la vie politique par un parti a annihilé ses fonctions matière électorale.

52 Après les élections présidentielles, le contexte sociopolitique évolue vers l'établissement d'une situation politique stable. C'est dans la plus grande discrétion que s'engagent des pourparlers entre des représentants du pouvoir et une partie du FRUD dans le pays et sans intervention extérieure. 
África (São Paulo, 1978, Online), São Paulo, n. 42, p. 177-219, 2021

blique, des textes sont publiés par la presse gouvernementale, signés par un groupe des dirigeants ${ }^{53}$, qui se proclament du FRUD. Après des contacts ont lieu en secret entre cette aile-modérée et des membres du gouvernement, qui aboutissent à un premier accord de paix signé le 26 décembre 1994, lequel a eu des conséquences politiques importantes, elles n'ont pas surpris les observateurs de la vie politique djiboutienne. En effet 6 mois après sa signature, le gouvernement est remanié ; un remaniement qui répond à un objectif politique du président Hassan Gouled et de son entourage : isoler davantage la direction du FRUD. Deux membres du FRUD-modéré, le Président et le Secrétaire Général, font leur entrée dans l'équipe gouvernementale ${ }^{54}$. Théoriquement cela paraît une nouvelle vision de la gestion politique dans ce pays après le régime de parti unique. La question est de savoir si les deux membres ex rebelles du gouvernement font intégrer au RPP ou vont créer leur propre parti. Sans doute résultant d'un calcul politique de l'élite du parti dominant, en mars 1996, un quatrième parti est légalisé sous la dénomination de FRUD dont le Président et le Secrétaire Général sont les deux ministres FRUD du gouvernement. Pourquoi ont-ils attendu deux années pour la légalisation de leur parti ? Contrairement à d'autres leaders de l'opposition, les nouveaux ministres n'interviennent pas dans la politique nationale dans les mêmes conditions. Le nouveau parti forme une coalition gouvernementale avec le parti gouvernemental, le RPP.

Le FRUD légalisé ne présente pas un candidat aux élections présidentielles d'avril 199, il apporte son soutien au candidat désigné par le RPP, Ismaël Omar Guelleh. Face à des difficultés financières et une législation qui favorise le parti et le candidat du pouvoir, les leaders des partis de l'opposition ne présentent pas leurs candidatures, mais ils soutiennent un candidat outsider, qui est un député dissident du RPP. Le candidat du pouvoir contrôle des ressources tant politiques (de l'appareil d'Etat et du parti gouvernemental) que socioéconomiques (connu par la population,

\footnotetext{
53 Ils prétendent être élus à la tête de la direction de la rébellion à la suite d'un congrès ; ils se démarquent de la direction et établissent des pourparlers avec le gouvernement. Elle est qualifiée d'aile modérée. Mais la direction officielle, dirigée par un ancien Premier ministre, feu Ahmed Dini conteste une telle prétention.

54 Mais cela n'est qu'une étape de ce long processus de fin de conflit armé. Les nouveaux ministres n'intègrent pas le parti gouvernemental, le RPP. Selon ledit accord, le Frud-mondéré est associé à la gestion des affaires du pays.
} 
La vie politique djiboutienne de 1992 a 2021 : quelques reflexions sur le changement et les reformes politiques

présenté comme l'homme de l'accord de décembre 1994, le contrôle des médias publics et disposant des moyens financiers importants) face à un député, inconnu par l'électorat national. Selon les résultats officiels publiés par le ministère de l'Intérieur, et confirmés par le Conseil Constitutionnel, le candidat gouvernemental remporte cette élection avec 74\%. Malgré la participation d'un autre candidat que celui du pouvoir, l'élection présidentielle d'avril 1999 ne peut être considérée comme une élection compétitive, parce que le candidat qui s'affrontait à celui de la coalition du pouvoir jouait le jeu apparent du pluralisme aux élections politiques dans ce pays; il ne dispose pas un appui institutionnel, financier et un appareil politique. Les élections présidentielles de 2005 ont été boycottées par l'opposition, le seul candidat était le président sortant, qui entamait son deuxième et dernier mandat à la présidence de la République. Les opposants critiquent les conditions d'organisation des élections et surtout le contrôle de l'administration par le candidat unique et des fraudes inévitables par exemple l'absence d'une encre indélébile, ce qui permet à une personne de voter à différents bureaux de vote et ainsi de gonfler exagérément le nombre de votants.

Elections présidentielles du 9 avril 1999

\begin{tabular}{|l|l|}
\hline \multicolumn{1}{|c|}{ Candidats } & \multicolumn{1}{c|}{ Pourcentage des votes } \\
\hline Ismaël Omar Guelleh & $74,09 \%$. \\
\hline Moussa Ahmed Idriss & $25,78 \%$ \\
\hline
\end{tabular}

Sources: ministère de l'Intérieur. Elaboration personnelle.

Moussa Ahmed Idriss est un homme politique des années 1960, indépendantiste et infirmier de profession. Elu à l'Assemblée Nationale, membre du parti unique. Il est passé à l'opposition. Il a été le candidat des partis de l'opposition. 
Elections présidentielles du 7 mai 2005

\begin{tabular}{|c|c|}
\hline \multicolumn{1}{|c|}{ Candidats } & Pourcentage des votes \\
\hline Ismaël Omar Guelleh & $96,85 \%$ \\
\hline
\end{tabular}

Sources : ministère de l'Intérieur. Elaboration personnelle.

C'est une élection qui rappelle le régime de parti unique, donc de candidat unique, présenté par le parti gouvernemental. Critiquant les conditions d'organisation, le refus du gouvernement d'actualiser le recensement électoral et le consensus sur la constitution des cartes électorales, mais aussi pour le financement de la compagne électorale, chère selon les leaders de l'opposition, ces derniers ne prennent pas part aux élections. Le candidat de la coalition du pouvoir se présentait seul à sa propre succession, ce qui enlevait tout enjeu à ce scrutin.

Elections présidentielles du 8 avril 2011

\begin{tabular}{|l|l|}
\hline \multicolumn{1}{|c|}{ Candidats } & \multicolumn{1}{c|}{ Pourcentage des votes } \\
\hline Ismaël Omar Guelleh & $80.63 \%$ \\
\hline Mohamed Warsama Ragueh & $19,37 \%$ \\
\hline
\end{tabular}

Sources: ministère de l'Intérieur. Elaboration personnelle.

Le président sortant se présentait pour un troisième mandat après la révision constitutionnelle de 2010. Les partis de l'opposition, opposés à cette réforme, n'ont pas présenté un ou des candidats. Son concurrent, Mohamed Warsama Ragueh, est un candidat libre. C'est un ancien magistrat, novice en politique. Il est accusé par les leaders de l'opposition de fair-play pour des élections dont l'issu n'avait pas de doute. Le candidat du pouvoir est déclaré vainqueur. A la différence des acteurs politiques, il a reconnu sa défaite électorale.

Elections présidentielles du 9 avril 2021

\begin{tabular}{|l|c|}
\hline \multicolumn{1}{|c|}{ Candidats } & Pourcentage des votes \\
\hline Ismaël Omar Guelleh & $97,3 \%$ \\
\hline Zakaria Ismael Farah & $2,7 \%$ \\
\hline
\end{tabular}

Sources: ministère de l'Intérieur. Elaboration personnelle.

Le Président sortant se présentait pour un $5^{\text {ème }}$ mandat consécutif. Zakaria Ismaël Farah est un candidat indépendant, inconnu du public djiboutien. 
La vie politique djiboutienne de 1992 a 2021 : quelques reflexions sur le changement et les reformes politiques

Il a vécu à l'étranger, il est un entrepreneur. Ce n'est pas la première fois qu'un candidat indépendant se présente, mais la première fois qu'un candidat, qui n'a été un haut fonctionnaire. Il a créé le Mouvement pour le Développement et l'Equilibre de la Nation Djiboutienne (MDEND). Il n'a pas vraiment mené une compagne électorale.

\subsection{LE CONTRÔLE DE L'ORGANISATION DES ÉLECTIONS}

Comme on a pu analyser dans le paragraphe antérieur, le processus électoral a été sous contrôle total du ministère de l'Intérieur, donc du pouvoir, qui est à la fois juge et parti, quant à l'organisation administrative, temporelle (la date des élections) et la proclamation des résultats de toutes les compétitions électorales. Les leaders des partis de l'opposition ont demandé en 1992 la création d'une institution compétente dans ce domaine, dont les membres seraient élus ou désignés par les partis politiques.

Ce n'est qu'en 2002 que, par décret présidentiel, est créée une Commission Electorale Nationale Indépendante $\left(\mathrm{CENI}^{55}\right)$ pour l'organisation des élections. Théoriquement la supervision de celles-ci est l'objet de celle nouvelle institution. Mais elle n'a pas compétence pour la supervision des élections municipales et régionales ${ }^{56}$. Si son existence même paraît être une évolution du nouveau système politique, elle n'est pas reçue de la même manière par l'opposition et le pouvoir, surtout quant à sa composition. C'est une vielle demande de l'opposition, mais celle-ci conteste sa composition ${ }^{57}$,

55 Le décret $\mathrm{n}^{\circ}$ 2002-0198/PR/MID porte composition et fonctionnement de la Commission Electorale Nationale Indépendante.

56 La constitution du 15 septembre 1992 a repris un des points de la constitution du 27 juin dernier - qui n'a jamais été adopté - la création des régions. La nouvelle constitution institutionnalise la division du pays en municipalités et régions dont les conseils sont élus. Mais ce n'est qu'en 2002 que la loi de la décentralisation a été votée et les premières élections municipales et régionales interviennent en 2006. L'opposition s'est peu mobilisée. Par contre, des mouvements politiques locaux de création récente ont présenté des candidates/s. Mais là aussi on assiste une victoire de la coalition du pouvoir.

57 Selon l'art. 3 Décret $n^{\circ}$ 2002-0198/PR/MID portant composition et fonctionnement de la Commission Electorale Nationale Indépendante, elle est composée de 13 personnes à Commission Electorale Nationale Indépendante (pour le District de Djibouti) désignées de la manière suivante : 3 par le gouvernement, 3 par le Président de l'Assemblée Nationale, 3 magistrats (ayant au moins 6 ans d'expérience) et 3 représentants de la société civile et 1 représentant par parti politique. Quant aux districts, le nombre total est de 7 le mode de désignation est le même. 
qui selon elle, est placée sous le contrôle du pouvoir. En effet son président est nommé par le gouvernement. Au niveau de chaque district il est créé des commissions régionales.

En effet sur le plan institutionnel, la compétition est mieux encadrée par rapport au régime de parti unique. Aussi elle permet la participation de tous les acteurs politiques. Mais comment peut-elle être indépendante du pouvoir en place? Que signifie l'indépendance d'une institution où il y a une surreprésentation des personnes nommées par les institutions contrôlées par le parti gouvernemental ? Ce n'est pas une institution permanente ; ses membres sont nommés à chaque élection. Au moins symboliquement par leur présence dans l'institution, les partis de l'opposition ont accès à certaines informations, ce qui change par rapport à la situation antérieure.

Aussi sur le plan symbolique, quelques mois avant cette échéance électorale, l'Assemblée Nationale adopte la Loi n 192/AN/02/4ème L le 13 novembre 2002, qui institue un système de quota dans les fonctions électives et dans l'administration de l'Etat. En fait il s'agit d'une politique de promotion de la femme sur le plan de la participation politique active. Il est recommandé aux partis politiques présentant des candidats à ces élections à inclure des femmes sur leurs listes. Mais sans attendre cette loi, le Parti du Renouveau Démocratique (PRD) l'a déjà fait en présentant une candidate dans sa liste pour les premières élections pluralistes de décembre 1992. Cette décision découle d'une décision du président de la République, élu en avril 1999 et non d'un changement de valeurs sociales de la population. Il a nommé dans son premier gouvernement une femme, la première à un poste ministériel, ministre déléguée auprès du Premier Ministre, Chargée de la Promotion de la Femme, du Bien Etre familial et des Affaires Sociales. La décision d'inclure un pourcentage de femmes dans les listes électorales fait suite à une loi adoptée le 7 juillet 2002 (Loi n 173/AN/02/4ème L) relative à une politique nationale en matière d'intégration de la femme dans le développement. Les 7 candidates présentées par la coalition gouvernementale, l'UMP, ont été élues. Et pour la première est élue une députée de l'opposition lors des dernières élections législatives en février 2013.

Par la régularité des élections législatives et présidentielles on assiste au moins à une institutionnalisation du pouvoir, laquelle est une forme pacifique de conquérir le pouvoir. Mais toutes ces élections sont remportées par l'ex 
La vie politique djiboutienne de 1992 a 2021 : quelques reflexions sur le changement et les reformes politiques

parti unique et ses associés ; elles sont contestées par les partis de l'opposition pour fraudes et irrégularités matérielles. Elles font l'objet de recours pour annulation au conseil constitutionnel, mais aucun n'a aboutit à l'annulation d'aucune d'elles.

Les défaites successives mais aussi les victoires faciles marquent les partis politiques. Tous traversent à un moment ou à un autre de son évolution une crise «existentielle».

\subsection{LA CRISE DES PARTIS POLITIQUES: L'ENJEU DE LA DÉMOCRATIE DES FORMATIONS PARTISANES}

L'éclatement d'une crise n'est pas en soi un signe particulièrement préoccupant pour la vie des partis politiques au contraire ce n'est qu'une étape de leur vie (naissance, croissance et disparition). Par contre la solution à la crise révèle le type de fonctionnement interne et le niveau de démocratie du parti en question.

En République de Djibouti le parti a servit dès le début de sa création comme un instrument de contrôle de son principal dirigeant, qui est aussi son créateur. C'est une structure importante que tout entrepreneur politique doit contrôler dans sa carrière politique les ressources politiques rares. C'est dans cet esprit qu' est née le RPP en mars 1979 ; il a permit à Hassan Gouled Aptidon d'asseoir un contrôle sur la vie politique nationale et de s'entourer des hommes politiques qui le soutiennent. Dans un contexte de néopatrimonialisme, le parti politique sert à la fois un instrument idéologique qui appuie par exemple la construction de l'unité nationale et un moyen de contrôle social en s'insérant dans la société. Mais un parti unique n'évite pas l'affrontement des ambitions personnelles. Et à un moment donné, son fondateur pour une raison essentiellement d'âge ne peut être un arbitre entre les courants personnels qui s'affrontent au sein de la formation. N'étant pas un parti démocratique, la succession de son chef est toujours problématique.

A Djibouti la succession du président de la République est ouverte depuis quelques années et l'un des moyens pour succéder ce dernier est le contrôle des ressources politiques et symboliques du RPP. Comme toute structure humaine de pouvoir, des courants s'opposent pour le contrôle de 
África (São Paulo, 1978, Online), São Paulo, n. 42, p. 177-219, 2021

la direction du parti ; des alliances entre membres influents se forment. Le président a un contrôle encore sur la vie du parti et aucun signe apparent de crise n'est perceptible. Or tout cela change avec l'évolution du contexte sociopolitique ou l'accélération de «l'histoire locale». Un des actes qui est à la base de la révélation de la crise dont l'opinion publique est le remaniement ministériel de juin 1995. Comme on a pu démontrer plus haut l'objectif poursuivi est de faire place au président et au secrétaire général de l'aile modérée du FRUD au sein du gouvernement. Mais ceci a créé une opposition de certains membres du gouvernement et du RPP. Ainsi le Secrétaire Général du RPP, et ancien ministre des Affaires Etrangères et de la Coopération Internationale, Moumin Bahdon Fareh est muté au ministère de la Justice, des Affaires Musulmanes et Plénipotentiaires ; il montre une réticence à occuper ce nouveau portefeuille ministériel. Son refus montre la division interne du parti ancienne que l'opinion nationale n'a jamais eu connaissance avant cette date. Proche collaborateur du président de la République depuis les années 1970, il est considéré comme l'un de ses successeurs. Sa nomination à ce ministère est interprétée comme une manière de réduire ses chances de le succéder un jour. Parmi ses concurrents, on trouve Ismaël Guedi Hared et Ismaël Omar Guelleh, respectivement Directeur de Cabinet et Chef de Cabinet de la Présidence. L'une des conséquences de cette crise est le report du congrès du RPP.

Mais les crises n'affectent pas de la même manière et n'ont pas les mêmes conséquences pour tous les partis politiques djiboutiens ; elles affaiblissent plus les nouveaux partis que l'ancien parti unique. Ses membres influents contrôlent d'importantes ressources politiques et socioéconomiques au sens large du terme incluant la justice, laquelle est utilisée pour mettre fin à une crise du parti gouvernemental. La division interne des nouvelles forces politiques fragilise davantage des structures en formation, qui n'ont pas une base sociale solide. Ce sont des crises politiquement exploitées par le parti dominant par l'attribution du récépissé de reconnaissance d'une nouvelle direction, qui est une stratégie du parti gouvernemental. Aussi les leaders contestés ont peu de ressources politiques et sociales pour affronter des échéances politiques importantes et de fait les partis perdent une partie de leur électoral. 
La vie politique djiboutienne de 1992 a 2021 : quelques reflexions sur le changement et les reformes politiques

\section{LES RÉFORMES CONSTITUTIONNELLES ET ÉLECTORALES : DÉVELOPPEMENT OU FREIN AU PROCESSUS DÉMOCRATIQUE.}

Il faut distinguer deux types de réformes, celles qui renforcent le pouvoir en place, et surtout son chef, et celles qui marquent une avancée vers plus de démocratie, par exemple en instaurant d'autres voies de participation citoyenne. Dans l'histoire constitutionnelle et politique de l'Afrique francophone, on peut observer que certaines révisions constitutionnelles constituent des sources tensions sociopolitiques ${ }^{58}$. Ces révisions poursuivent différents objectifs, d'une part le renforcement du pouvoir exécutif et d'autre part le contrôle du pouvoir législatif.

\subsection{CONTEXTE DES RÉVISIONS CONSTITUTIONNELLES}

A la fin de la décennie 1980, il y a eu ce qu'on a appelé les réformes constitutionnelles que beaucoup d'africanistes ont confondu avec la transition démocratique alors que la fin de la décennie 1990 on assiste à des révisions constitutionnelles. De la même manière qu'il y a eu une euphorie de modifier la constitution, il y a eu aussi une tendance de la part de beaucoup de présidents de réviser le texte suprême quelques mois avant la fin de leurs seconds mandats. Qu'est-ce qu'une révision ? Réviser n'est pas adopter une nouvelle constitution, mais une modification limitée de certaines dispositions de la constitution existante. S'appuyant sur des auteurs constitutionnels, Amed Laurent Franck DIAO la définit «la modification du contenu de la Constitution en procédant à l'adoption d'une loi constitutionnelle, selon les dispositions prévues par la Constitution en vigueur et précisées par les textes relatifs à la révision ${ }^{59}$ ». Mais

58 Bahdon Mohamed Abdillahi, Des modifications constitutionnelles, source de tensions sociopolitiques, AfriquEducation, ${ }^{\circ} 485 \mathrm{H}$, février 2020, pp.16-17.

59 Amed Laurent Franck DIAO, LES REVISIONS CONSTITUTIONNELLES EN AFRIQUE : CRITIQUE EN LEGALITE ET LEGITIMITE, 2018-2019, in https://www.google.com/ search?q=les $+\mathrm{r} \% \mathrm{C} 3 \%$ A9visions + constitutionnelles+en+Afrique+noire\&ei=OAVGYZ6ZFMWvkwXdwI-YDg\&oq=les+r\%C3\%A9visions+constitutionnelles+en+Afrique+noire\&gs_lcp=Cgdnd3Mtd216EAMyCAghEBYQHRAeMggIIRAWEB0QHjoHCAAQsAMQQzoICAAQgAQQsAM6EAguEMcBENEDEMgDELADEEM6CwguEIAEEMgDELADOhEILhCABBDHARDRAxDIAxCw AzoKCC4Qy AMQs AMQQzoRCC4QgAQQxwEQrwEQyAMQsAM6BAgAEEM6CwguEIAEEMcBENEDOgUIABCABDoLCC4QgAQQxwEQowI6BQguEIAEOgcILhBDEJMCOgQILhBDOgoILhDHARCjAhB- 
África (São Paulo, 1978, Online), São Paulo, n. 42, p. 177-219, 2021

cette définition prête à confusion, malgré le respect dispositions prévues par la constitution pour sa révision, il y a l'adoption d'une loi constitutionnelle. L'auteur reconnaît que «le canevas de modification de ces normes, surtout constitutionnelles, pour s'ajuster à la société et en même temps résister aux manipulations $^{60}$ est assez souvent sujet à discussion ${ }^{61} \gg$. La discussion a été déclenchée non seulement par le moment choisi par le pouvoir de réviser le texte constitutionnel en vigueur, mais aussi par les dispositions visées. L'une d'elles est l'article qui limite le nombre de mandats présidentiels à deux. Mais l'inquiétude n'est pas la révision en soi, mais leur légalité et leur légimité.

ANDZOKA Séverin, "Les bizarreries constitutionnelles comme moyen de refus de l'alternance démocratique en Afrique », in SOMA Abdoulaye (dir.), Alternances politiques en Afrique : Défis démocratiques et enjeux constitutionnels, Actes du colloque international de la SBDC, Ouagadougou, éd. Rônier-Tikanson, 2016, pp.323-339.

DIOP El Hadj Oumar, L'instrumentalisation de la Constitution dans les régimes politiques africains, Dakar, éd. CREDILA/OVIPA, 2017

Des révisions interviennent au Cameroun en 1996 et en 2008, en Côte d'Ivoire en 2016 et en 2020, au Tchad en 2003 et 2016, au Togo en 2002 et en 2015. Ces révisions ont lieu dans des pays où la transition du régime de parti unique au système multipartisan a échoué. Ce sont des dirigeants de partis uniques ont gouverné ces pays, à part un coup d'Etat et une courte transition en Côte d'Ivoire avec l'élection de l'opposant, Laurent Gbagbo. En fait, l'objectif principal de ces révisions n'était autre que la suppression de la limitation à deux mandats successifs, introduite dans les premières constitutions adoptées après les mouvements populaires de contestation. Elle fut considérée comme une nouveauté politique. Or des présidents et des dirigeants poli-

DOgsILhCABBDHARCvAToOCC4QgAQQxwEQowIQkwI6BwgAEIAEEAo6AggmOgYIABAWEB46DgguEIAEEMcBEK8BEJMCOg4ILhCABBDHARDRAxCTAjoICC4QgAQQkwI6BAgAEBM6CAgAEBYQHhATOggIABANEB4QEzoECCEQCkoFCDgSATFKBAhBGAFQxRJYzlhg-FpoBXAAeACAAYoBiAHYJJIBBTQwLjEzmAEAoAEByAEPwAEB\&sclient=gws-wiz\&ved=0ahUKEwielZvp6ojzAhXF16QKHV3gA-MQ4dUDCA4\&uact $=5$.

60 Matthieu FAU-NOUGARET « Manipulations constitutionnelles et coup d'Etat constitutionnel en Afrique francophone », Afrilex, janvier 2016, pp 1-18.

61 Idem, Amed Laurent Franck Dialo. 
La vie politique djiboutienne de 1992 a 2021 : quelques reflexions sur le changement et les reformes politiques

tiques d'une culture politique différente à l'esprit démocratique de respect des normes constitutionnelle, cette disposition a été la première à être prise en compte par les auteurs des nouvelles réformes avant la fin du second et dernier mandat présidentiel. En la supprimant, c'est la pérennisation au pouvoir, qui est institutionnalisée, et donc de rendre encore difficile l'alternance au sommet de l'Etat.

Le nouveau régime politique djiboutien a suivi cette mode. Et c'est en l'an 2010 que le gouvernement adopte par la voie parlementaire une réforme constitutionnelle. A l'instar des gouvernements de ces pays, celui de la République de Djibouti supprime la disposition constitutionnelle sur la limitation des mandats d'un président en exercice. En République de Djibouti, il y a eu plusieurs révisions constitutionnelles en 2006, 2008 et 2010.

\subsection{RENFORCEMENT DU RÉGIME PRÉSIDENTIEL}

La constitution du 15 septembre 1992 avait repris le même régime présidentialiste des lois constitutionnelles de 1977. De 1977 à 1992, le président de la République était à la fois Chef de l'Etat et chef du gouvernement. Le bicaméralisme de l'exécutif n'était qu'un symbole repris de la constitution de la $5{ }^{\text {ème }}$ République française. Le président adoptait des ordonnances alors qu'à partir de 1992. Il y a une certaine normalisation d'un parlement qui acquiert des compétences. Reprenant le modèle français, les articles 57 et 58 de la constitution du 15 septembre 1992 marquent des domaines d'intervention de la loi, donc du pouvoir législatif, et du pouvoir réglementaire, donc du pouvoir exécutif.

Mais à la lecture des articles 58 et 59 , on observe un renforcement des actes et pouvoirs du président de la République. Ainsi à la demande de ce dernier, des textes de forme législative adoptés par le pouvoir législative, peuvent être modifiés par Conseil Constitutionnel et deviennent de la compétence du pouvoir réglementaire. Et par l'article 59, le Président de la République partage l'initiative parlementaire avec les membres de l'Assemblée Nationale. Comme les députés, il a aussi le droit à l'amendement. Par contre, il est le seul à déterminer la politique de la nation. Il dispose du pouvoir réglementaire (art.30 de la constitution). Le président de la République est donc 
à la fois un acteur du pouvoir législatif et du pouvoir exécutif. Le seul acte où l'Assemblée Nationale a le dernier mot est lors de la déclaration d'une guerre (art.68). Mais c'est purement formel, parce que la décision est prise par le Président de la République après consultation de ses conseillers en défense et les généraux de l'armée nationale.

Les rapports entre les deux pouvoirs politiques sont déséquilibrés au profit du détenteur du pouvoir exécutif. Comme les autres membres du gouvernement, il est responsable des actes accomplis dans l'exercice de ses fonctions, mais en cas de haute trahison selon l'article 84 de la constitution. C'est un progrès par rapport aux premières lois constitutionnelles. Mais le problème est comment déterminer non seulement la trahison, mais la haute trahison. Aucune disposition constitution, ni une loi ne déterminent ce qu' est la haute trahison alors que les membres du gouvernement, c'est-à-dire les ministres, ministres délégués et Secrétaires d'Etat peuvent être poursuivis.es «pénalement responsables des actes accomplis dans l'exercice de leurs fonctions et qualifiés crimes ou délits au moment où ils ont été commis » (art.8). La mise en accusation relève des membres de l'Assemblée Nationale. Or une Assemblée, contrôlée par la coalition du pouvoir, peut-elle mettre en accusation des membres du gouvernement pour des faits délictuels ?

\subsection{LE CONTRÔLE DU POUVOIR LÉGISLATIF}

Le contrôle du pouvoir législatif par le pouvoir exécutif est présenté par les constitutionnalistes ou les juristes en droit public comme la rationalisation du parlementarisme. Mais rationalisation répond à un déséquilibre des pouvoirs de l'Etat au profit du législatif quand le Président de la République était élu par le Parlement. La vie politique djiboutienne n'a pas connu un tel contexte.

Le contrôle du pouvoir législatif n'est pas seulement le fait du pouvoir exécutif et de son détenteur, mais aussi de l'opposition politique parlementaire ou qui veut renter au parlement. L'autre réforme, attendue par l'opposition, est celle relative à la loi électorale pour l'élection des parlementaires. Comme on a pu analyser antérieurement, c'est en 1992, quelques mois avant les premières élections pluralistes, qu'a été adaptée par une loi organique relative 
La vie politique djiboutienne de 1992 a 2021 : quelques reflexions sur le changement et les reformes politiques

lalégislation électorale ${ }^{62}$.

Théoriquement cette dernière réforme a introduit une dose de proportionnalité pour la représentation des partis au pouvoir. Selon le ministre de l'Intérieur de l'époque l'introduction de la dose de proportionnelle «émane de la volonté politique du Président de la République», qui serait «un geste d'ouverture politique ${ }^{63}$ ».

Mais si la proportionnalité peut-être considérée comme une certaine justice quant à la représentation d'une partie de l'électorat, qui a voté pour des petits partis, celle-ci poursuit un autre objectif ; elle est manipulée. Ses auteurs décident une proportionnalité qui les favorise, puisque $80 \%$ des sièges revient au parti qui a eu le plus de suffrages exprimés; même si la différence de votés exprimés est minime comme cela s'est produit lors des dernières élections législatives en février 2013. Et donc il n'y aurait pas une nécessité d'aller à un deuxième tour, ce qui a existé théoriquement dans l'ancienne loi électorale. Comme toujours la coalition du pouvoir est proclamée gagnante (par le ministère de l'Intérieur), ce que conteste l'opposition ${ }^{64}$ et dépose un recours en annulation au conseil constitutionnel. Suivant sa jurisprudence depuis 1993, cette institution rejette sa requête pour non respect de délai de recours.

Dans toute transition il y a apparition de nouvelles institutions et des réformes constitutionnelles, judiciaires et politiques et sociales. Elles marquent une évolution de la société, qui passe d'un stade à un autre dans un processus de changement imparable quand les conditions sont réunies, même s'il n'y pas vraiment un consensus. Dans la majorité des cas celles-ci reflètent un certain mimétisme institutionnel et même politique (débat politique) aux systèmes politiques étrangers, que ça soit l'ex métropole coloniale ou les modèles récents des pays africains.

Dans le contexte politique et la culture politique djiboutienne, il est rare qu'un parlement contrôlé par l'opposition. Non seulement par la législation,

62

63 Entretien du Journal La Nation du 6 décembre 2012

64 En plus du recours qu'elle a introduit, l'opposition appelle à ses membres à manifester contre les résultats. Ses leaders ont été arrêtées, jugés et emprisonnés. Celles et ceux qui ont été élues/s ont refusé de siéger à l'Assemblée Nationale, et ont même allées jusqu'à constituer, selon eux un «parlement élu démocratiquement.» 
África (São Paulo, 1978, Online), São Paulo, n. 42, p. 177-219, 2021

mais aussi par la recourt à la répression, le gouvernement s' est doté les moyens pour avoir une majorité parlementaire.

\section{CONCLUSION}

Le président Hassan Gouled ne pourrait maintenir pendant longtemps son refus de «suivre» l'effet de «mode» à propos du processus dénommé assez rapidement de transition démocratique. Le processus de changement politique de septembre 1992 avec un référendum constitutionnel, marquant ainsi une étape important de l'histoire politique de la République de Djibouti : l'adoption des valeurs libérales tout au moins théoriquement, il se fait par étapes et son évolution est placée sous le contrôle du pouvoir en place. Sur ce point c'est une reprise d'autres situations africaines par exemple comme celle du Burkina Faso, du Gabon, du Zaïre où le pouvoir présidentiel a impulsé un processus qui n'a pas échappé à son contrôle. On assiste à un changement superficiel.

Si l'adoption d'une constitution reconnaissant le pluralisme politique est une étape importante dans la vie politique djiboutienne, elle n'est qu'une variable parmi tant d'autres quant à l'instauration d'abord et ensuite la consolidation d'un nouveau régime politique pluraliste et ouvert, même les éléments caractéristiques du régime antérieur n'ont pas disparu par exemple la répression contre les opposants. Ce nouveau régime se matérialise par un ensemble de facteurs tant légaux donc théoriques que pratiques comme l'institution d'une nouvelle pratique politique et surtout d'une compétition électorale ouverte. De 1992 à 2005, on assiste à un développement institutionnel avec l'apparition de nouvelles institutions politiques, judiciaires et de médiation dont le but théorique affirmé est l'institution d'une démocratie participative, d'un gouvernement démocratique et la recherche du dialogue entre les acteurs sociopolitiques du pays. Or en analysant la constitution et surtout la répartition des pouvoirs politique on remarque la permanence d'une certaine culture politique : la concentration du pouvoir étatique entre les mains du premier personnage de l'Etat, le Chef de l'Etat. Malgré l'existence d'un Premier ministre et compte tenu des changements sociaux depuis 1977, le président de la République concentre le pouvoir exécutif, et en plus il partage l'initiative parlementaire avec les députés, qui relative la séparation des pouvoirs. 
La vie politique djiboutienne de 1992 a 2021 : quelques reflexions sur le changement et les reformes politiques

Or la concentration de ce pouvoir risque de poser un problème grave dans les années à venir si l'Assemblée Nationale passe sous la majorité d'un parti ou d'une coalition de partis de l'opposition, c'est l'apparition d'une hypothétique cohabitation entre une majorité parlementaire et un président d'un autre parti. Jusqu'à présent et grâce à un système électoral avantageux pour le parti gouvernemental et ses coalitions, les élections pluralistes n'ont pas introduit un changement dans de la configuration politique la chambre parlementaire. Aussi aucune réforme du système électoral n'est à l'ordre du jour. Cependant l'existence d'une coalition parlementaire, regroupant 4 partis, mais dont les bases ne partagent pas toutes les mêmes programmes ou n'ont pas la même attente (entre un parti gouvernemental contrôlant l'administration et des partis politiques nouveaux), peut conduire à des oppositions internes, lesquelles peuvent à leur tour conduire à l'apparition d'une opposition au sein de l'assemblée durant une législature. Donc on se trouverait dans une situation particulière où une opposition naît des rivalités entre les leaders des partis d'une coalition parlementaire et non pas par la voie électorale. Une des causes d'une crise de cette coalition serait la difficulté pour le parti dominant à redistribuer les ressources dont il contrôle. La rupture de la coalition peut-elle conduire à l'organisation d'élections anticipées ? C'est une option qu'on ne peut exclure.

Quant à la pratique politique de ces trente dernières années, elle ne correspond pas au nouveau discours sur le respect des droits de l'homme et de la démocratie. Confondant victoire électorale et gestion d'une nouvelle situation sociopolitique, marquée par la permanence d'une crise économique et sociale, accentuée par le Programme d'Ajustement Structurel signé en 1994 avec le FMI, le pouvoir privilégie une gestion autoritaire des conflits sociaux et politiques. Il y a une contradiction entre un régime qui se veut démocratique et la répression politique et sociale dont subissent des membres de l'opposition et des acteurs.es de la société civile. La gestion de cette crise mobilise très peu de gens et très peu d'organisations ; il faut noter que les structures de la société civile sont encore faibles et dans une situation de crise socioéconomique elles ne peuvent mobiliser que très peu de personnes. Cette manière de gérer politiquement les problèmes sociopolitiques est renforcée par une tentative de coup d'Etat avortée en décembre 2000 par l'augmentation de la répression et l'obsession de la sécurité personnelle du chef de l'Etat. 
Le système institutionnel a changé depuis 1992, mais ce n'est pas un changement de fonctionnement d'un système politique, hérité de l'époque du parti unique. Dans le cas djiboutien, le changement politique introduit en 1991 est un mécanisme politique contrôlé par le maintien de l'élite gouvernante, composée principalement des acteurs des dernières années de l'époque coloniale. Celle-ci a tenté de s'adapter aux exigences du nouveau contexte politique et économique tant du continent africain qu'au niveau mondial : promotion des droits de l'homme, la question du genre ou des femmes et l'organisation d'élections pluralistes. Or la démocratie ne se décrète pas, elle dépend d'un ensemble d'actes et de pratiques. L'organisation des élections pluralistes n'en est qu'une d'elles.

La période de changement politique initié en 1992 n'a pas comblé les attentes sociales, ni l'espoir de nombreux acteurs politiques et sociaux du pays, ni en matière de «bonne gouvernance» et ni en matière de développement économique et social... Si la coalition de partis peut-être considérée comme une évolution de la politique intérieure dans la mesure où elle permet des négociations entre différentes formations, son existence depuis 1997 n'a pas introduit une nouvelle culture politique et des nouvelles pratiques, qui favorisent la participation et la transparence aux questions de la cité. Jusqu'à nos jours il n'y a pas de commission d'enquête à l'Assemblée Nationale sur des questions importantes qui toucheraient la gestion du gouvernement ou de certains de ses membres. Sur ce point, le parlementarisme djiboutien est limité dans ses compétences.

La nouvelle polarité du système politique de 1992, pouvoir/opposition, n'a pas eu lieu. En observant les faits et les réformes avec le recul nécessaire entre 1992 et 2021 pour comprendre vraiment, on pourrait même être amené à affirmer que rien n'a changé réellement.

\section{BIBLIOGRAPHIE}

\section{ARTICLES DE REVUES}

Bayart J.-F., «La problématique de la démocratie en Afrique noire : La Baule, et puis après?». Politique africaine (43), oct. 91 : p. 5-20. 
La vie politique djiboutienne de 1992 a 2021 : quelques reflexions sur le changement et les reformes politiques

Banegas, Richard, «Action collective et transition politique en Afrique : la Conférence nationale du Bénin.» Cultures et conflits (17), print. 1995 : p. 137-175.

Clapham, Christopher, Democratisation in Africa: obstacles and prospects. Third World Quarterly 14 (3), 1993, p. 423-438.

Coquery-Vidrovitch, C., La politique en Afrique noire : héritage et avenir. Genre humain (26), aut. 92-hiv. 93 : p. 119-140.

Decalo, S., The process, prospects and constraints of democratization in Africa. African Affairs (London) 91 (362), janv. 92: p. 7-35.

Decraene, Phillipe, «Réflexions sur l'exigence africaine de démocratisation», Défense nationale, $\mathrm{n}^{\circ} 48$ (10), octobre 92: p. 123-136.

Ebo, H., «Djibouti, Tensions sociopolitiques sur fond de succession», L'Afrique Politique Revendications populaires et recompositions politiques, Paris, Karthala, 1997, pp.85-109.

Galloy Martine-Renée et Gruénais Marc-Éric, «Des dictateurs africains sortis des urnes», Novembre 1997, pp. 12-13

Manga, P., Réflexions sur la dynamique constitutionnelle en Afrique. Revue juridique et politique, indépendance et coopération (1), janv.-avr. 1994 : p. 46-70.

Marchal R., «Mitterrand, Djibouti et la Corne de l'Afrique», Politique africaine, juin 1995

M'Bembe A., «Diagnostic sur les dérapages de la transition démocratique en Afrique», Afrique 2000, Entretien, février 1993 : p.

Mbembe, A., «Traditions de l'autoritarisme et problèmes de gouvernement en Afrique sub-saharienne», Africa Development 17 (1), 1992 : p. 37-64.

Médard J.F., «Autoritarismes et démocraties en Afrique noire», Politique africaine $\mathrm{N}^{\circ} 4$. oct. $1991:$ p..

Medhanie T., «Les modèles de transition démocratique», Afrique 2000, août 1993. pp.

Michalon Thierry, «Pour la suppression de l'élection présidentielle en Afrique», Le Monde Diplomatique, janvier 1998, pp. 24-35

Anyang Nyongo, P. «Instabilité politique et perspectives de démocratie en Afrique», Politique étrangère, 1988, pp. 589 - 601

\section{Livres}

Adamon, Afize D., Le renouveau démocratique au Bénin : la Conférence nationale des Forces vives et la période de transition. - Paris, Harmattan, 1995 : 223 p.

Aden R., Djibouti, Clef de la Mer Rouge, Paris, Editions Caractères, 1986.

Akindes F., Les mirages de la démocratie en Afrique subsaharienne francophone, Paris, Any- 
África (São Paulo, 1978, Online), São Paulo, n. 42, p. 177-219, 2021

ang Nyongo P., (Dir), Afrique : la longue marche vers la démocratie : Etat autoritaire et résistances populaires, Parism Publisud, 1988.

Karthala, 1996.

Badie, B, L'Etat importé, l'occidentalisation de l'ordre politique, Paris, Fayard, 1992.

Bakary Akin, Tessy D., Des militaires aux avocats : une autre forme de coup d'État, la «Conférence Nationale Souveraine «. Géopolitique africaine 15 (2), sept.-oct. 92.

Bayart J-F., et al, La politique par le bas en Afrique : contribution à une problématique de la démocratie, Paris, Khartala, 1992.

Boulaga F., Les conférences nationales en Afrique noire : une affaire à suivre, Paris, Karthala, 1993.

Coubba A, Djibouti, une nation en otage, Paris, L'Harmattan, 1993.

Djibouti : enjeux politiques et rivalités politique, Paris, L'Harmattan, 1994.

Conac, G. L'Afrique en transition vers le pluralisme politique, Paris, Economica, 1993.

Fukuyama, F., El fin de la Historia y el último hombre, Barcelona, Planeta, 1992.

Gouad Farah., La République de Djibouti : naissance d'un Etat, chronologie, Djibouti, Imprimerie Nationale, 1982.

Held, David, La democracia y el orden global: del estado moderno al gobierno cosmopolita, Barcelona, Paidós, 1997

Hermet G., Sociologie de la construction démocratique, Paris, Economica, 1986. Le désenchantement de la liberté, Paris, Fayard, 1993.

Des élections pas comme les autres (en collaboration avec J.J. Linz et A. Rouquié), Paris, Fondation Nationale de Science Politique, 1978.

Houmed I. I., Indépendance, démocratie, enjeux stratégiques à Djibouti, Paris, l'Harmattan, 2002.

Hugo Ph. et Hugot P., Histoire de la République. Des origines à la République, Paris, L'Harmattan, 1985.

Huntington, S. La tercera ola: la democratización a finales del siglo XX., Barcelona, Paidós, 1994.

Kalu N. Kalu, «Intégrer la démocratie et le développement en Afrique : l'impératif du capital institutionnel», Revue Internationale des Sciences Administratives, Vol. 70, N³, sept. 2004, pp.569-5870

Lamarche G., «La fin des beaux jours à Djibouti», Politique Étrangère, été, 1993. 
La vie politique djiboutienne de 1992 a 2021 : quelques reflexions sur le changement et les reformes politiques

Lavroff G.-D., Les partis politiques en Afrique Noire, Paris, Presse Universitaire de France, 1970 .

Lochouan Y., Les Afar de la Corne de l'Afrique, Mémoire de DEA, Université Bordeaux I, 1982.

Joseph E., «Le renouvellement politique en Afrique subsaharienne : Un défi pour les années 1990», Afrique 2000, novembre 1990 : p.15-20.

Mohamed A., Ourron-Djibouti 1991-1994 : du maquis afar à la paix de braves, Paris, Paris, L'Harmattan, 2002.

Mohamed Abdillahi Bahdon, «La juridiction constitutionnelle en République de Djibouti : enjeux et perspectives de la garantie du respect des droits et des principes fondamentaux.», Cuestiones Constitucionales, Revista Electrónica de la Universidad Nacional Autónoma de México, Julio-Diciembre de 2006, p.3-32.

Mohamed Abdillahi Bahdon, "El acceso la justicia como paradigma de democratización en la República de Yibuti", Papeles del Grupo de Estudios Africanos, Documento 2, noviembre de 2005, Universidad Autónoma de Madrid.

Monkotan K., «Des modes originaux d'expression démocratique en Afrique», Afrique 2000, Novembre 1990.

Morel, Yves, «Démocratisation en Afrique noire : les « conférences nationales». Études 376 (6), juin 1992, pp. 733-743.

Morlino, L. Como cambian los regimenes, Madrid, Centro de Estudios Constitucionales, 1985

Moukoko M., «Pierre, Régimes militaires et transition démocratique en Afrique : à la recherche d'un cadre d'analyse théorique», Afrique 2000 (Paris) (13), avr.-juin 1993, pp. 39-58.

Ndiaye, Tafsir Malick, «Des transitions démocratiques en Afrique», Alternative démocratique dans le Tiers Monde (6), juil.-déc. 1992, pp. 13-29.

Otayeck R., Djibouti : construction nationale et contraintes stratégiques, CEAN, Bordeaux, 1985.

Otayeck R., La démocratie entre mobilisations identitaires et besoin d'Etat : y a-t-il une "exception" africaine? Autrement, No, 1999.

Pridham, Geoffrey, “The International Dimension of democratisation: theory, practice and inter-regional comparisons" pp.7-28 in Geoffrey Pridham, Eric Herring and George Sanford, Building Democracy? The International Dimension of Democratisation? Leicester. Leicester University Press, 1997.

Otayek, René «Démocratie, culture politique, sociétés plurales: une approche comparative à partir de situations africaines», Revue française de science politique, 47 (6), décembre, 1997, 
África (São Paulo, 1978, Online), São Paulo, n. 42, p. 177-219, 2021

p. 798-822.

...........»L'universel et le culturel en démocratie», Pyramide. Revue internationale d'analyse et de prospective (Ouagadougou), 1, avril, 1997 p. 35-45.

«Oyono D., «Du parti unique au multipartisme : environnement international et processus de démocratisation en Afrique», Afrique 2000, Août 1991, p. ??

Quantin, Patrick et al, Les transitions démocratiques africaines, Paris, Karthala, 1996.

Raynal, Jean-Jacques, «Les conférences nationales en Afrique : au-delà du mythe, la démocratie ?» Penant 104 (816), oct.-déc. 94 Doctrine: p. 310-321.

Sall, E., «Bicéphalisme du pouvoir exécutif dans les régimes politiques d'Afrique noire : crises et mutations», Pénant, sept.déc. 1997, p. ? ?

Sandbwok, Richard, 1996, "Transitions without consolidations: democratization in six African cases", Third World Quartely, 17, pp.69-87

Sindjoun Luc et Owona Mathias Eric, Politisation du droit et juridicisation de la politique : l'esprit sociopolitique du droit à la transition démocratique au Cameroun. En Du Bois de Gaudusson Jean et Darbon Dominique, La création du droit en Afrique, Paris, Karthala, 1997

Toulabor, Comy, «La transition démocratique en Afrique», Afrique 2000 (4), fév. 91 : p. 55-70.

Zaïki Laïki, Un monde privé de sens, Paris, Seuil, 1994.

Zolberg, R., "L'influence des facteurs "externes" sur l'ordre politique interne", En Grawitz, M. et Lecas, J., (Dir.), Traité de Science Politique, T1: L'ordre politique, Presses Universitaires de France, 1985, p.567-597.

\section{ARticles de JOURNAUX}

La Nation (journal gouvernemental de la République de Djibouti),

Politique Africaine, La Corne de l'Afrique, Nº juin 1995.

Le Progrès (journal du parti gouvernemental djiboutien).

Le Renouveau (journal du PRD).

La République (journal du PND).

Jeune Afrique, Supplément Djibouti Juillet 1990.

Loi n¹92/AN/02/4ème L le 13 novembre 2002 